MATHEMATICS OF COMPUTATION

Volume 67, Number 223, July 1998, Pages 1253-1283

S 0025-5718(98)00952-1

\title{
DETECTING PERFECT POWERS IN ESSENTIALLY LINEAR TIME
}

\author{
DANIEL J. BERNSTEIN
}

\begin{abstract}
This paper (1) gives complete details of an algorithm to compute approximate $k$ th roots; (2) uses this in an algorithm that, given an integer $n>1$, either writes $n$ as a perfect power or proves that $n$ is not a perfect power; (3) proves, using Loxton's theorem on multiple linear forms in logarithms, that this perfect-power decomposition algorithm runs in time $(\log n)^{1+o(1)}$.
\end{abstract}

\section{INTRODUCTION}

An integer $n>1$ is a perfect power if there are integers $x$ and $k>1$ with $n=x^{k}$. Note that $k \leq \log _{2} n$; also, the minimal $k$ is prime.

A perfect-power detection algorithm is an algorithm that, given an integer $n>1$, figures out whether $n$ is a perfect power. A perfect-power decomposition algorithm does more: if $n$ is a perfect power, it finds $x$ and $k>1$ with $n=x^{k}$. A perfect-power classification algorithm does everything one could expect: it writes $n$ in the form $x^{k}$ with $k$ maximal.

Theorem 1. There is a perfect-power classification algorithm that uses time at most $\left(\log _{2} n\right)^{1+o(1)}$ for $n \rightarrow \infty$.

A more precise bound is $\left(\log _{2} n\right) \exp (O(\sqrt{\log \log n \log \log \log n}))$ for $n>16$.

This paper is organized as a proof of Theorem 1. Part I reviews integer and floating-point arithmetic. Part II develops an algorithm to compute $k$ th roots. Part III presents a perfect-power decomposition algorithm, Algorithm X. It bounds the run time of Algorithm X in terms of a function $F(n)$. Part IV and Part V analyze $F(n)$. Part $\mathrm{V}$ completes the proof of Theorem 1 by showing that $F(n)$ is essentially linear in $\log n$. Part VI surveys several practical improvements.

Motivation. Before attempting to factor $n$ with algorithms such as the number field sieve [16], one should make sure that $n$ is not a perfect power, or at least not a prime power. This is a practical reason to implement some power-testing method, though not necessarily a quick one.

Speed is more important in other applications. According to [18] there is a theoretically interesting method of finding all small factors of $n$ (to be presented in a successor to [19]) for which perfect-power classification can be a bottleneck.

Received by the editor October 11, 1995 and, in revised form, April 10, 1997.

1991 Mathematics Subject Classification. Primary 11Y16; Secondary 11J86, 65G05.

This paper was included in the author's thesis at the University of California at Berkeley. The author was supported in part by a National Science Foundation Graduate Fellowship.

(C)1997 Daniel J. Bernstein 
See $[4$, section 1] for another example. Here average performance, for $n$ chosen randomly from a large interval, is more important than worst-case performance. See Part IV for results on the average performance of Algorithm X.

For readers who want to compute high-precision inverses and roots. One of $\mathrm{my}^{1}$ major tools is of independent practical interest. Section 8 gives complete theoretical and practical details of an algorithm to compute $y^{-1 / k}$ to $b$ bits. My goal here was to produce something immediately useful in practice.

For readers interested in transcendental number theory. One of my major tools is of independent theoretical interest. Section 19 contains a corrected proof of a bound on the number of perfect powers in a short interval. Both the bound and the corrected proof are due to Loxton; the underlying theorem about linear forms in logarithms is also due to Loxton. Sections 16, 17, 18, and 19 may be read independently of the rest of the paper.

Index of notation. $d(i, j) \S 9 ; \operatorname{div}_{b}(r, k) \S 5 ; \operatorname{div}_{2, b}(r, k) \S 21 ; F(n) \S 12 ; H(\alpha) \S 17 ;$ $\vartheta(t) \S 14 ; \vartheta_{2}(t) \S 14 ; \ell(t) \S 14 ; M(b) \S 3 ; \mu(b) \S 3 ; \operatorname{mul}(r, k) \S 4 ; \operatorname{mul}_{2, b}(m, k) \S 21 ;$ $\operatorname{nroot}_{b}(y, k) \S 8 ; \operatorname{nroot}_{2, b}(y, k) \S 21 ; P(k) \S 6 ; \operatorname{pow}_{b}(r, k) \S 6 ; \operatorname{pow}_{2, b}(x, k) \S 21 ; \operatorname{round} t$ $\S 10 ; \operatorname{trunc}_{b} r \S 5$.

\section{Acknowledgments}

Many thanks to Hendrik W. Lenstra, Jr., John Loxton, Michael Parks, Igor Shparlinski, Jon Sorenson, and the referee for their helpful comments. Lenstra pointed out that the method of Part III had a $p$-adic analogue; Shparlinski suggested that [20] would help in the $F(n)$ analysis; Loxton supplied the corrected proof of [20, Theorem 1] shown in section 19.

\section{PART I. ARITHMETIC}

\section{Integer ARIthmetic}

I represent a positive integer $n$ inside a computer as a string of bits giving $n$ 's binary expansion. It is easy to add and subtract integers in this form.

A $b$-bit number is a positive integer smaller than $2^{b}$. The string representing such an integer has at most $b$ bits.

The algorithms in this paper use a black box that performs integer multiplication. By definition $M$-time is the time spent inside this black box. Let $M(b)$ be an upper bound on the time used by this black box to compute the product of two $b$-bit numbers. In this paper, I show that the $M$-time used by various algorithms is bounded by a sum of various $M(b)$ 's.

Define $\mu(b)=\max \{M(j) / j: 1 \leq j \leq b\}$. Then $\mu(b)$ is a nondecreasing function of $b$, with $M(b) \leq b \mu(b)$. If $M(b) / b$ is already nondecreasing then $\mu(b)=M(b) / b$.

Lemma 3.1. If $K(t) \in t^{o(1)}$ and $L(v)=\max \{K(t): t \leq v\}$ then $L(v) \in v^{o(1)}$.

So if $M(b) \in b^{1+o(1)}$ then $\mu(b) \in b^{o(1)}$. When $\mu(b) \in b^{o(1)}$ the black box is performing fast multiplication.

\footnotetext{
${ }^{1}$ I have abandoned the practice of mechanically replacing "I" with "we" in my papers.
} 
Proof. Fix $\epsilon>0$. Select $u>1$ such that $-\epsilon \lg t<\lg K(t)<\epsilon \lg t$ for $t>u$. Fix $v>\max \left\{u, L(u)^{1 / \epsilon}\right\}$. If $t \leq u$ then $K(t) \leq L(u)$ so $\lg K(t) \leq \lg L(u)<\epsilon \lg v$; if $u<t \leq v$ then $\lg K(t)<\epsilon \lg t \leq \epsilon \lg v$. Hence $\lg L(v)<\epsilon \lg v$. On the other hand $\lg L(v) \geq \lg K(v)>-\epsilon \lg v$.

Notes. Theorem 1 refers to time, not $M$-time. For definiteness I select a RAM, with logarithmic cost for memory access, as a somewhat realistic model of computation; the black box can be implemented using any fast multiplication algorithm. The reader may then verify that the time spent by these algorithms is almost exclusively $M$-time. The same is true in practice.

See [15, section 4.3.3] for a discussion of fast multiplication algorithms. For the best known bounds on multiplication speed see [29] or [1]; note that it is possible to build a different algorithm, achieving the same bounds, out of the method of [24].

\section{Floating-Point ARithmetic}

A positive floating-point number is a positive integer divided by a power of 2 . The computer can store a pair $(a, n)$, with $a$ an integer and $n$ a positive integer, to represent the positive floating-point number $2^{a} n$. (In practice $|a|$ is always very small, so $a$ can be stored as a machine integer.)

Notice that $(a, n)$ and $(a-1,2 n)$ represent the same number. The computer can shift among representations. It could repeatedly divide $n$ by 2 until it is odd, for example, to make the representation unique.

Let $(a, n)$ and $\left(a^{\prime}, n^{\prime}\right)$ represent the positive floating-point numbers $r=2^{a} n$ and $r^{\prime}=2^{a^{\prime}} n^{\prime}$ respectively. Set $f=\min \left\{a, a^{\prime}\right\}$. Then $r+r^{\prime}=2^{f}\left(2^{a-f} n+2^{a^{\prime}-f} n^{\prime}\right)$ is represented by the pair $\left(f, 2^{a-f} n+2^{a^{\prime}-f} n^{\prime}\right)$. Similarly, if $r>r^{\prime}$, then $r-r^{\prime}$ is a positive floating-point number represented by the pair $\left(f, 2^{a-f} n-2^{a^{\prime}-f} n^{\prime}\right)$.

Multiplication is easier: $\left(a+a^{\prime}, n n^{\prime}\right)$ represents the product $r r^{\prime}$. If $n$ and $n^{\prime}$ are both $b$-bit numbers then the $M$-time here is at most $M(b)$.

Define $\operatorname{mul}(r, k)=k r$ for $r$ a positive floating-point number and $k$ a positive integer. To compute $\operatorname{mul}(r, k)$ I use an algorithm designed for multiplying by small integers; time spent computing mul is not $M$-time.

Notes. A typical computer has hardware designed to handle a finite set of floatingpoint numbers. One may study the extent to which operations on the real numbers can be approximated by operations on such a small set [15, section 4.2.2]; the difference is called "roundoff error." Rewriting $2^{a-1}(2 n)$ as $2^{a} n$ is often called "denormalization"; it is rarely considered useful.

My point of view is somewhat different. I do not worry too much about computer hardware, and I do not work within any particular finite set. I regard approximation not as causing "error" but as limiting the precision used for intermediate operations, thus speeding up the computation. I can work with $n$ more efficiently than $2 n$, so I happily rewrite $2^{a-1}(2 n)$ as $2^{a} n$.

A floating-point number is also known as a dyadic rational [23, page 435].

See [15, exercise 4.3.1-13] for an algorithm to compute $\operatorname{mul}(r, k)$.

\section{FloAting-POint truncation}

In this section, I define truncation to $b$ bits, written trunc $b$, and show that $r /$ trunc $_{b} r$ is between 1 and $1+2^{1-b}$. More generally, for any positive integer $k, \mathrm{I}$ 
define $\operatorname{div}_{b}(r, k)$ as a floating-point approximation to $r / k$, so that $r / k \operatorname{div}_{b}(r, k)$ is between 1 and $1+2^{1-b}$.

Fix $b \geq 1$. Set $\operatorname{div}_{b}(a, n, k)=\left(a+f-\lceil\lg k\rceil-b,\left\lfloor n / 2^{f-\lceil\lg k\rceil-b} k\right\rfloor\right)$, where $2^{f-1} \leq n<2^{f}$. (Note that $f-\lceil\lg k\rceil-b$ may be negative.) This map induces a map, also denoted $\operatorname{div}_{b}$, upon positive floating-point numbers:

$$
\operatorname{div}_{b}\left(2^{a} n, k\right)=2^{a+f-\lceil\lg k\rceil-b}\left\lfloor n / 2^{f-\lceil\lg k\rceil-b} k\right\rfloor \quad \text { if } 2^{f-1} \leq n<2^{f} .
$$

To compute $\operatorname{div}_{b}(r, k)$ I use an algorithm designed for dividing by small integers; time spent computing $\operatorname{div}_{b}$ is not $M$-time.

Lemma 5.1. Fix $b \geq 1$ and $k \geq 1$. Let $r$ be a positive floating-point number, and set $s=\operatorname{div}_{b}(r, k)$. Then $s \leq r / k<s\left(1+2^{1-b}\right)$.

Proof. Put $r=2^{a} n$ and define $f$ by $2^{f-1} \leq n<2^{f}$. Also write $g=f-\lceil\lg k\rceil-b$ and $m=\left\lfloor n / 2^{g} k\right\rfloor$, so that $s=2^{a+g} m$. Then $m \leq n / 2^{g} k<m+1$; furthermore $m \geq\left\lfloor 2^{f-1} / 2^{g} k\right\rfloor=\left\lfloor 2^{\lceil\lg k\rceil} 2^{b-1} / k\right\rfloor \geq\left\lfloor 2^{b-1}\right\rfloor=2^{b-1}$. Thus $2^{a+g} m \leq 2^{a} n / k<$ $2^{a+g}(m+1)=2^{a+g} m(1+1 / m) \leq 2^{a+g} m\left(1+2^{1-b}\right)$. So $s \leq r / k<s\left(1+2^{1-b}\right)$ as desired.

Define $\operatorname{trunc}_{b} r=\operatorname{div}_{b}(r, 1)$; i.e., $\operatorname{trunc}_{b} 2^{a} n=2^{a+f-b}\left\lfloor n / 2^{f-b}\right\rfloor$ if $2^{f-1} \leq n<2^{f}$. Observe that $\left\lfloor n / 2^{f-b}\right\rfloor$ is a $b$-bit number.

Lemma 5.2. Fix $b \geq 1$. Let $r$ be a positive floating-point number, and set $s=$ trunc $_{b} r$. Then $s \leq r<s\left(1+2^{1-b}\right)$.

Proof. Take $k=1$ in Lemma 5.1 .

Notes. For most computers a base such as $2^{32}$ is more convenient than base 2 . It is tempting to replace trunc by a function that keeps a few extra bits "up to the word boundary." One may safely succumb to this temptation, as long as $M(b)$ is also changed appropriately: the crucial properties of trunc are Lemma 5.2 and the fact that two values of $\operatorname{trunc}_{b}$ may be multiplied in $M$-time $M(b)$.

See [15, exercise 4.3.1-16] for an algorithm to compute $\operatorname{div}_{b}(r, k)$.

\section{Approximate powers}

Let $r$ be a positive floating-point number, and let $k$ and $b$ be positive integers. Then $\operatorname{pow}_{b}(r, k)$, the $b$-bit approximate $k$ th power of $r$, is a floating-point approximation to $r^{k}$. In this section, I show how to compute $\operatorname{pow}_{b}(r, k)$ in $M$-time at most $P(k) M(b)$, where $P(k) \leq 2\lfloor\lg k\rfloor$.

Define $P(k)$ for $k \geq 1$ as follows: $P(1)=0 ; P(2 k)=P(k)+1 ; P(2 k+1)=$ $P(2 k)+1$.

Lemma 6.1. $P(k) \leq 2\lfloor\lg k\rfloor$.

Proof. For $k=1, P(k)=0$ and $\lg k=0$. If $P(k) \leq 2\lfloor\lg k\rfloor$ then $P(k)+2 \leq$ $2\lfloor\lg k\rfloor+2=2\lfloor\lg 2 k\rfloor$, so $P(2 k)=P(k)+1<P(k)+2 \leq 2\lfloor\lg 2 k\rfloor$ and $P(2 k+1)=$ $P(k)+2 \leq 2\lfloor\lg 2 k\rfloor \leq 2\lfloor\lg (2 k+1)\rfloor$.

Define $\operatorname{pow}_{b}(r, k)$ for $k \geq 1$ as follows:

$$
\begin{aligned}
\operatorname{pow}_{b}(r, 1) & =\operatorname{trunc}_{b} r \\
\operatorname{pow}_{b}(r, 2 k) & =\operatorname{trunc}_{b}\left(\operatorname{pow}_{b}(r, k)^{2}\right), \\
\operatorname{pow}_{b}(r, 2 k+1) & =\operatorname{trunc}_{b}\left(\operatorname{pow}_{b}(r, 2 k) \operatorname{pow}_{b}(r, 1)\right) .
\end{aligned}
$$


Lemma 6.2. $\operatorname{pow}_{b}(r, k) \leq r^{k}<\operatorname{pow}_{b}(r, k)\left(1+2^{1-b}\right)^{2 k-1}$.

Proof. If $k=1$ then $\operatorname{trunc}_{b} r \leq r<\left(\operatorname{trunc}_{b} r\right)\left(1+2^{1-b}\right)$ by Lemma 5.2. If $k>1$ there is, by definition of pow, some partition $i+j=k$ with $\operatorname{pow}_{b}(r, k)=$ $\operatorname{trunc}_{b}\left(\operatorname{pow}_{b}(r, i) \operatorname{pow}_{b}(r, j)\right)$. If $\operatorname{pow}_{b}(r, i) \leq r^{i}<\operatorname{pow}_{b}(r, i)\left(1+2^{1-b}\right)^{2 i-1}$ and $\operatorname{pow}_{b}(r, j) \leq r^{j}<\operatorname{pow}_{b}(r, j)\left(1+2^{1-b}\right)^{2 j-1}$ then $\operatorname{pow}_{b}(r, k) \leq \operatorname{pow}_{b}(r, i) \operatorname{pow}_{b}(r, j) \leq$ $r^{i} r^{j}<\operatorname{pow}_{b}(r, i) \operatorname{pow}_{b}(r, j)\left(1+2^{1-b}\right)^{2(i+j)-2}<\operatorname{pow}_{b}(r, k)\left(1+2^{1-b}\right)^{2 k-1}$.

Algorithm $P$. Given a positive floating-point number $r$ and two positive integers $b, k$, to print $\operatorname{pow}_{b}(r, k)$ :

1. If $k=1$, print trunc $b$ and stop.

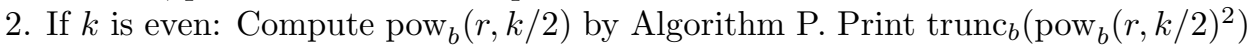
and stop.

3. Compute $\operatorname{pow}_{b}(r, k-1)$ by Algorithm P. Print trunc $b\left(\operatorname{pow}_{b}(r, k-1) \operatorname{trunc}_{b} r\right)$.

Lemma 6.3. Algorithm $P$ computes $\operatorname{pow}_{b}(r, k)$ in $M$-time at most $P(k) M(b)$.

Proof. Count the number of multiplications. There are $0=P(1)$ multiplications for $\operatorname{pow}_{b}(r, 1)$. If there are at most $P(k)$ multiplications for $\operatorname{pow}_{b}(r, k)$, then there are at most $P(k)+1=P(2 k)$ multiplications for $\operatorname{pow}_{b}(r, 2 k)$, and at most $P(2 k)+1=$ $P(2 k+1)$ multiplications for $\operatorname{pow}_{b}(r, 2 k+1)$.

Notes. Algorithm $\mathrm{P}$ is the left-to-right binary method, which comes from a broad class of powering algorithms indexed by addition chains [15, section 4.6.3]. Lemma 6.2 would remain true if $\operatorname{pow}_{b}(r, k)$ were replaced with the output from any algorithm in this class. For many $k$ one can find an algorithm using fewer than $P(k)$ multiplications; this is useful in practice. See [15, section 4.6.3] and [11, section 1.2 ] for further discussion.

For large $k$ it is probably better to compute $r^{k}$ as $\exp (k \log r)$ by the methods of [9], which take essentially linear time.

\section{PART II. ROOTS}

\section{Some OVERly SPECIFIC INEQUALITIES}

Lemma 7.1. If $\kappa>0$ and $0<\epsilon<1$ then $(1+\epsilon / 4 \kappa)^{2 \kappa}<1+\epsilon$.

Lemma 7.2. If $\kappa \geq 1$ then $7 / 8 \leq(1-1 / 8 \kappa)^{\kappa}$.

Lemma 7.3. If $0<t<1 / 36$ then $(1+3 t)(1+t)(1+32 t / 3)<1+16 t$.

Lemma 7.4. If $\kappa \geq 1$ and $0<t<1 /(4 \kappa+4)$ then $(1+t)^{2 \kappa+3}-1<16 t(7 \kappa-2) / 9$.

\section{Approximate roots}

In this section, I consider the problem of root extraction: computing $y^{1 / k}$, given a positive floating-point number $y$ and a positive integer $k$. I also consider the problem of inversion: computing $y^{-1}$. I solve both problems by showing how to compute $y^{-1 / k}$. Then $y^{1 / k}=y\left(y^{-1 / k}\right)^{k-1}$. (Alternatively $y^{1 / k}=\left(y^{-1 / 1}\right)^{-1 / k}$; more generally $y^{1 / k}=\left(y^{-1 / k_{1}}\right)^{-1 / k_{2}}$ if $k=k_{1} k_{2}$.)

For each positive integer $b$, I will construct a floating-point number $\operatorname{nroot}_{b}(y, k)$ satisfying $\operatorname{nroot}_{b}(y, k)\left(1-2^{-b}\right)<y^{-1 / k}<\operatorname{nroot}_{b}(y, k)\left(1+2^{-b}\right)$. My method, in brief, is a binary search for small $b$, and then Newton's method with increasing precision for all larger $b$. 
Binary search: the idea. I am trying to find a root $z$ of $z^{k} y-1$. Binary search means guessing the bits of $z$, one by one. Given an interval $R$ surrounding the root, I evaluate $z^{k} y-1$ at the midpoint of $R$. Depending on the sign of the answer, I replace $R$ by either the left half of $R$ or the right half of $R$. I repeat until $R$ is sufficiently small.

To speed up the computation, I only approximate $z^{k} y-1$. If the answer is too close to 0 for me to be sure about its sign, I replace $R$ with the middle half of $R$.

Binary search: the algorithm. For $b \leq 3+\lceil\lg k\rceil$, I define and construct $\operatorname{nroot}_{b}(y, k)$ by Algorithm B below. For the time spent by Algorithm B, see Lemma 8.1. For the accuracy of its output, see Lemma 8.3.

A brief comment on the constant 993/1024 in Algorithm B: The proof of Lemma 8.2 will use the fact that $993 / 1024$ is between $32 / 33$ and $e^{-1 / 33}$. It is the "simplest" floating-point number in this range.

Algorithm B. To compute $\operatorname{nroot}_{b}(y, k)$ for $1 \leq b \leq\lceil\lg 8 k\rceil$ : In advance, find the exponent $g$ satisfying $2^{g-1}<y \leq 2^{g}$, and set $a=\lfloor-g / k\rfloor$, so that $2^{a} \leq y^{-1 / k}<$ $2^{a+1}$. Also set $B=\lceil\lg (66(2 k+1))\rceil$.

1. Set $z \leftarrow 2^{a}+2^{a-1}, j \leftarrow 1$.

2. (See Lemma 8.2 for an invariant.) Now $\operatorname{nroot}_{j}(y, k)=z$. If $j=b$, stop.

3. Compute $r \leftarrow \operatorname{trunc}_{B}\left(\operatorname{pow}_{B}(z, k) \operatorname{trunc}_{B} y\right)$.

4. If $r \leq 993 / 1024$, set $z \leftarrow z+2^{a-j-1}$.

5. If $r>1$, set $z \leftarrow z-2^{a-j-1}$.

6. Set $j \leftarrow j+1$. Go back to step 2 .

Lemma 8.1. For $b \leq\lceil\lg 8 k\rceil$, Algorithm $B$ computes $\operatorname{nroot}_{b}(y, k)$ in $M$-time at most $(b-1)(P(k)+1) M(\lceil\lg (66(2 k+1))\rceil)$.

Proof. Algorithm B gets to $\operatorname{nroot}_{b}(y, k)$ in $b-1$ iterations. Each iteration takes time at most $P(k) M(B)$ to find pow $_{B}(z, k)$, by Lemma 6.3 , and time at most $M(B)$ to multiply by $\operatorname{trunc}_{B} y$.

Lemma 8.2. $2^{a} \leq z-2^{a-j} \leq y^{-1 / k}<z+2^{a-j} \leq 2^{a+1}$ at step 2 of Algorithm B.

Proof. Induct on $j$. For $j=1: z-2^{a-1}=2^{a} \leq y^{-1 / k}<2^{a+1}=z+2^{a-1}$.

Assume the result true for $j$. Then, at step 3, the computation of $r$ gives $r \leq$ $z^{k} y<r\left(1+2^{1-B}\right)^{2 k+1}$ by Lemma 5.2 and Lemma 6.2 . But $\left(1+2^{1-B}\right)^{2 k+1}<$ $(1+1 /(33(2 k+1)))^{2 k+1}<e^{1 / 33}<1024 / 993$ so $r \leq z^{k} y<r(1024 / 993)$.

Case 1: $r>1$. Then $z^{k} y>1$. Algorithm B replaces $z$ by $z^{\prime}=z-2^{a-j-1}$; and $z^{\prime}-2^{a-j-1}=z-2^{a-j} \leq y^{-1 / k}<z=z^{\prime}+2^{a-j-1}$.

Case 2: $r \leq 993 / 1024$. Then $z^{k} y<(993 / 1024)(1024 / 993)=1 ; z$ is replaced by $z^{\prime}=z+2^{a-j-1}$, and $z^{\prime}-2^{a-j-1}=z<y^{-1 / k}<z+2^{a-j}=z^{\prime}+2^{a-j-1}$.

Case 3: $993 / 1024<r \leq 1$. Then Algorithm B leaves $z$ unchanged. I have $j \leq\lceil\lg 8 k\rceil-1<\lg 8 k$ so $2^{-j-2} \geq 1 / 32 k$. Thus $\left(1+2^{-j-2}\right)^{k} \geq 1+1 / 32$, so

$$
\left(z+2^{a-j-1}\right)^{k} y=z^{k} y\left(1+\frac{2^{a-j-1}}{z}\right)^{k}>z^{k} y\left(1+\frac{2^{a-j-1}}{2^{a+1}}\right)^{k}>\frac{993}{1024} \frac{33}{32}>1 .
$$

On the other hand $\left(1-2^{-j-2}\right)^{k}<32 / 33$. So

$$
\left(z-2^{a-j-1}\right)^{k} y=z^{k} y\left(1-\frac{2^{a-j-1}}{z}\right)^{k}<z^{k} y\left(1-\frac{2^{a-j-1}}{2^{a+1}}\right)^{k}<\frac{1024}{993} \frac{32}{33}<1 .
$$

Hence $z-2^{a-j-1}<y^{-1 / k}<z+2^{a-j-1}$ as desired. 
Lemma 8.3. $\operatorname{nroot}_{b}(y, k)\left(1-2^{-b}\right)<y^{-1 / k}<\operatorname{nroot}_{b}(y, k)\left(1+2^{-b}\right)$ for $b \leq\lceil\lg 8 k\rceil$.

Proof. $\operatorname{nroot}_{b}(y, k)$ appears as $z$ in step 2 of Algorithm B when $j=b$. By Lemma $8.2,2^{a} \leq z-2^{a-b} \leq y^{-1 / k}<z+2^{a-b} \leq 2^{a+1}$. Then $z 2^{-b}>2^{a-b}$ so $z\left(1-2^{-b}\right)<$ $z-2^{a-b} \leq y^{-1 / k}<z+2^{a-b}<z\left(1+2^{-b}\right)$.

Newton's method: the idea. I am trying to find a root of $h(z)=z^{-k} y^{-1}-1$. Newton's method is to replace the first guess, $z$, by a much better guess, $z-$ $h(z) / h^{\prime}(z)=z+\left(z-y z^{k+1}\right) / k$. I repeat until $z$ has the desired accuracy.

Newton's method roughly doubles the number of correct digits on each iteration. To speed the computation, I compute the full-precision answer only on the last step; I work with only $1 / 2$ the digits in the previous step, $1 / 4$ in the step before that, and so on.

Newton's method: the algorithm. For $b \geq 4+\lceil\lg k\rceil$, I define and construct $\operatorname{nroot}_{b}(y, k)$ by Algorithm $\mathrm{N}$ below. For the $\operatorname{accuracy}_{\text {of }} \operatorname{nroot}_{b}(y, k)$, see Lemma 8.7. For the time spent by Algorithm N, see Lemma 8.4.

Algorithm $N$. To compute $\operatorname{nroot}_{b}(y, k)$ for $b \geq\lceil\lg 8 k\rceil+1$ : In advance set $b^{\prime}=$ $\lceil\lg 2 k\rceil+\lceil(b-\lceil\lg 2 k\rceil) / 2\rceil$ and $B=2 b^{\prime}+4-\lceil\lg k\rceil$. Note that $b^{\prime}<b$.

1. Compute $z \leftarrow \operatorname{nroot}_{b^{\prime}}(y, k)$, by Algorithm B if $b^{\prime} \leq\lceil\lg 8 k\rceil$ or by Algorithm $\mathrm{N}$ if $b^{\prime} \geq\lceil\lg 8 k\rceil+1$.

2. Set $r_{2} \leftarrow \operatorname{mul}\left(\operatorname{trunc}_{B} z, k+1\right)$.

3. Set $r_{3} \leftarrow \operatorname{trunc}_{B}\left(\operatorname{pow}_{B}(z, k+1) \operatorname{trunc}_{B} y\right)$.

4. Set $r_{4} \leftarrow \operatorname{div}_{B}\left(r_{2}-r_{3}, k\right)$. Now $\operatorname{nroot}_{b}(y, k)=r_{4}$.

Lemma 8.4. For $b \geq\lceil\lg 8 k\rceil+1$, Algorithm $N$ computes $\operatorname{nroot}_{b}(y, k)$ in $M$-time at most $T+K U$, where $T=\lceil\lg 4 k\rceil(P(k)+1) M(\lceil\lg (66(2 k+1))\rceil), K=P(k+1)+1$, and $U=(2 b+10+\lceil 8+\lg k\rceil\lceil\lg (b-\lceil\lg 2 k\rceil)-3\rceil) \mu(b+6)$.

Proof. $B-\lceil\lg 2 k\rceil-5=2\left(b^{\prime}-\lceil\lg 2 k\rceil\right)=2\lceil(b-\lceil\lg 2 k\rceil) / 2\rceil$, so $B$ is either $b+5$ or $b+6$. Hence $B+2 b^{\prime} \leq b+6+2\left(b^{\prime}-\lceil\lg 2 k\rceil\right)+2\lceil\lg 2 k\rceil \leq b+7+(b-\lceil\lg 2 k\rceil)+2\lceil\lg 2 k\rceil=$ $2 b+\lceil 8+\lg k\rceil$. Note that $\left\lceil\lg \left(b^{\prime}-\lceil\lg 2 k\rceil\right)\right\rceil=\lceil\lg (b-\lceil\lg 2 k\rceil)-1\rceil$.

Observe that $b^{\prime} \geq\lceil\lg 8 k\rceil$. If $b^{\prime}>\lceil\lg 8 k\rceil$, Algorithm $N$ calls itself to compute $\operatorname{nroot}_{b^{\prime}}(y, k)$. By induction, this call takes $M$-time at most $T+K U^{\prime}$, where $U^{\prime}=$ $\left(2 b^{\prime}+10+\lceil 8+\lg k\rceil\left\lceil\lg \left(b^{\prime}-\lceil\lg 2 k\rceil\right)-3\right\rceil\right) \mu\left(b^{\prime}+6\right)$.

If $b^{\prime}=\lceil\lg 8 k\rceil$, Algorithm N calls Algorithm B to compute $\operatorname{nroot}_{\lceil\lg 8 k\rceil}(y, k)$. By Lemma 8.1, this call takes $M$-time at most $T$. Note that in this case $U^{\prime}=0$, since $2\lceil\lg 8 k\rceil+10+\lceil 8+\lg k\rceil\lceil\lg (\lceil\lg 8 k\rceil-\lceil\lg 2 k\rceil)-3\rceil=0$.

So in either case step 1 of Algorithm $\mathrm{N}$ takes $M$-time at most $T+K U^{\prime}$. By Lemma 6.3, step 3 of Algorithm N uses $M$-time at most $P(k+1) M(B)+M(B)=$ $K M(B)$. Thus the total $M$-time used by Algorithm $\mathrm{N}$ is at most

$$
\begin{aligned}
T+ & K M(B)+K U^{\prime} \\
& =T+K M(B)+K\left(2 b^{\prime}+10+\lceil 8+\lg k\rceil\left\lceil\lg \left(b^{\prime}-\lceil\lg 2 k\rceil\right)-3\right\rceil\right) \mu\left(b^{\prime}+6\right) \\
& \leq T+K\left(B+2 b^{\prime}+10+\lceil 8+\lg k\rceil\left\lceil\lg \left(b^{\prime}-\lceil\lg 2 k\rceil\right)-3\right\rceil\right) \mu(b+6) \\
& \leq T+K\left(2 b+10+\lceil 8+\lg k\rceil+\lceil 8+\lg k\rceil\left\lceil\lg \left(b^{\prime}-\lceil\lg 2 k\rceil\right)-3\right\rceil\right) \mu(b+6) \\
& =T+K(2 b+10+\lceil 8+\lg k\rceil\lceil\lg (b-\lceil\lg 2 k\rceil)-3\rceil) \mu(b+6)=T+K U
\end{aligned}
$$

as claimed. 
Lemma 8.5. Define $w=\left((k+1) z-z^{k+1} y\right) / k$. If $z(1+\epsilon)=y^{-1 / k}$ and $\epsilon>-1 / 8 k$ then $w \leq y^{-1 / k} \leq w\left(1+4 k \epsilon^{2} / 3\right)$.

Proof. $1-k \epsilon \leq(1+\epsilon)^{-k}$, so $k+1-z^{k} y=k+1-(1+\epsilon)^{-k} \leq k+k \epsilon=k /\left(z y^{1 / k}\right)$, so $w=(z / k)\left(k+1-z^{k} y\right) \leq y^{-1 / k}$. Next $(1+\epsilon)^{k} \geq 1+k \epsilon \geq 1-1 / 8>1 / 2$, so $k+1 \geq 2>(1+\epsilon)^{-k}=z^{k} y$, so $w>0$. Finally

$$
\begin{aligned}
\frac{y^{-1 / k}}{w}-1 & =\frac{1-(1-k \epsilon)(1+\epsilon)^{k}}{(k+1)(1+\epsilon)^{k}-1}=\frac{1-(1-k \epsilon) /(k+1)}{(k+1)(1+\epsilon)^{k}-1}-\frac{1-k \epsilon}{k+1} \\
& \leq \frac{1-(1-k \epsilon) /(k+1)}{(k+1)(1+k \epsilon)-1}-\frac{1-k \epsilon}{k+1}=\frac{k \epsilon^{2}}{1+\epsilon+k \epsilon} \leq \frac{4}{3} k \epsilon^{2}
\end{aligned}
$$

since $1+(k+1) \epsilon \geq 1-(k+1) / 8 k \geq 1-1 / 4=3 / 4$.

Lemma 8.6. If $z\left(1-2^{-b^{\prime}}\right)<y^{-1 / k}<z\left(1+2^{-b^{\prime}}\right)$ in Algorithm $N$ then $r_{4}\left(1-2^{-b}\right)$ $<y^{-1 / k}<r_{4}\left(1+2^{-b}\right)$.

Proof. Define $w=\left((k+1) z-z^{k+1} y\right) / k$. The idea is that $w$ is very close to $y^{-1 / k}$, $\left(r_{2}-r_{3}\right) / k$ is very close to $w$, and $r_{4}$ is very close to $\left(r_{2}-r_{3}\right) / k$.

Define $\epsilon$ by $z(1+\epsilon)=y^{-1 / k}$, so that $-2^{-b^{\prime}}<\epsilon<2^{-b^{\prime}}$. Note that $b^{\prime} \geq\lceil\lg 8 k\rceil \geq$ $\lg 8 k$, so $2^{-b^{\prime}} \leq 1 / 8 k$, so $-1 / 8 k<\epsilon<1 / 8 k$.

$B$ is either $b+5$ or $b+6$, so $2^{5-B} \leq 2^{-b}$. Abbreviate $\delta=2^{1-B}$. Then $\delta<1 / 36$, $\delta<1 /(4 k+4)$, and $8(1+\delta)<9$. Also $(2 k) 2^{-2 b^{\prime}} \leq 2^{1+\lceil\lg k\rceil-2 b^{\prime}}=2^{5-B}=16 \delta$.

By construction $r_{2} \leq(k+1) z<r_{2}(1+\delta), r_{3} \leq z^{k+1} y<r_{3}(1+\delta)^{2 k+3}$, and $r_{4} \leq\left(r_{2}-r_{3}\right) / k<r_{4}(1+\delta)$.

By Lemma 7.2, $7 / 8 \leq(1-1 / 8 k)^{k}<(1+\epsilon)^{k}=y^{-1} z^{-k}$, so $z^{k} y<8 / 7$. So

$$
\frac{r_{3}}{r_{2}} \leq \frac{z^{k+1} y(1+\delta)}{(k+1) z}=\frac{z^{k} y(1+\delta)}{k+1}<\frac{8}{7} \frac{1+\delta}{k+1}<\frac{9}{7(k+1)}<\frac{2}{3} .
$$

By Lemma $8.5, w \leq y^{-1 / k}$, so

$$
\begin{aligned}
\frac{y^{-1 / k}}{r_{4}} & \geq \frac{w}{r_{4}} \geq \frac{k w}{r_{2}-r_{3}}=\frac{(k+1) z-z^{k+1} y}{r_{2}-r_{3}} \geq \frac{r_{2}-r_{3}(1+\delta)^{2 k+3}}{r_{2}-r_{3}} \\
& =1-\frac{(1+\delta)^{2 k+3}-1}{r_{2} / r_{3}-1}>1-\frac{(1+\delta)^{2 k+3}-1}{(7 k-2) / 9}>1-16 \delta
\end{aligned}
$$

by Lemma 7.4.

On the other hand, by Lemma $8.5, y^{-1 / k} \leq w\left(1+4 k \epsilon^{2} / 3\right)$, so

$$
\begin{aligned}
\frac{y^{-1 / k}}{r_{4}} & \leq \frac{w\left(1+4 k \epsilon^{2} / 3\right)}{r_{4}}<\frac{w\left(1+(2 / 3)(2 k) 2^{-2 b^{\prime}}\right)}{r_{4}} \leq \frac{w(1+(32 / 3) \delta)}{r_{4}} \\
& <\frac{k(1+\delta) w(1+(32 / 3) \delta)}{r_{2}-r_{3}}=\frac{(1+\delta)\left((k+1) z-z^{k+1} y\right)(1+(32 / 3) \delta)}{r_{2}-r_{3}} \\
& <\frac{(1+\delta)\left(r_{2}(1+\delta)-r_{3}\right)(1+(32 / 3) \delta)}{r_{2}-r_{3}} \\
& =(1+\delta)\left(1+\frac{32}{3} \delta\right)\left(1+\frac{\delta}{1-r_{3} / r_{2}}\right)<(1+\delta)\left(1+\frac{32}{3} \delta\right)(1+3 \delta) \\
& <1+16 \delta
\end{aligned}
$$

by Lemma 7.3 .

Lemma 8.7. $\operatorname{nroot}_{b}(y, k)\left(1-2^{-b}\right)<y^{-1 / k}<\operatorname{nroot}_{b}(y, k)\left(1+2^{-b}\right)$ for all $b$. 
Proof. For $b \leq\lceil\lg 8 k\rceil$ this is Lemma 8.3. For $b \geq\lceil\lg 8 k\rceil+1, \operatorname{nroot}_{b}(y, k)$ is $r_{4}$ in Algorithm N. By induction $z\left(1-2^{-b^{\prime}}\right)<y^{-1 / k}<z\left(1+2^{-b^{\prime}}\right)$, so $r_{4}\left(1-2^{-b}\right)<$ $y^{-1 / k}<r_{4}\left(1+2^{-b}\right)$ by Lemma 8.6.

Notes. Algorithms B and N are reasonably "tight": they do not use unnecessarily high precision. As the reader can see, I pay for this tightness in complex proofs, though the algorithms themselves are short and straightforward.

The basic outline of my method is well known, as is its approximate run time. For Newton's method with increasing precision see [9] (which popularized [8]) or [7, section 6.4]. For the specific case of inversion see also [15, Algorithm 4.3.3-R] or [1, page 282]. For a controlled number of steps of binary search as preparation for Newton's method see [4, section 3].

However, it is difficult to find a complete error analysis in the literature, let alone an algorithm carefully tuned for speed in light of such an analysis. An algorithm with results of unknown accuracy - or an algorithm not even stated explicitly - is of little value for implementors.

A notable exception for $k=1$ is [15, Algorithm 4.3.3-R], which is stated in full detail and supported by tight error bounds; but Algorithm $\mathrm{N}$ will be faster, because it pays close attention to the desired final precision.

For Newton's method generally see [26, section 9.4]. The inequalities in Lemma 8.5 follow from general facts of the form "when Newton's method is applied to the following class of nice functions, the iterates exhibit the following nice behavior."

Binary search as a root-finding technique is also known as bisection. For bisection generally see [26, section 9.1]. My use of binary search is closer in spirit to $[26$, section 9.1$]$ than to [4, section 3$]$ since I limit the precision of intermediate calculations.

For large $k$, just as $r^{k}$ is probably best computed as $\exp (k \log r), r^{1 / k}$ is probably best computed as $\exp ((\log r) / k)$ by the methods of [9].

\section{PART III. POWER TESTING}

\section{HOW TO TEST IF $n=x^{k}$}

Consider the problem of testing, given positive integers $n, x$, and $k$, whether $n=x^{k}$. I could simply compute $x^{k}$ and check whether it equals $n$. But I can eliminate most $(x, n)$ more efficiently, by checking whether $n=x^{k}$ is consistent with the first few digits of $n$ and $x$.

Algorithm C. Given positive integers $n, x, k$, to compute the sign of $n-x^{k}$ : In advance set $f=\lfloor\lg 2 n\rfloor$.

1. Set $b \leftarrow 1$.

2. Compute $r \leftarrow \operatorname{pow}_{b+\lceil\lg 8 k\rceil}(x, k)$.

3. If $n<r$, print -1 and stop.

4. If $r\left(1+2^{-b}\right) \leq n$, print 1 and stop.

5 . If $b \geq f$, print 0 and stop.

6. Set $b \leftarrow \min \{2 b, f\}$. Go back to step 2 .

The farther apart $n$ and $x^{k}$ are, the more quickly Algorithm $\mathrm{C}$ can tell them apart. Define a distance $d$ on integers as $d(i, j)=0$ when $i=j, d(i, j)=\lfloor\lg |i-j|\rfloor$ when $i \neq j$; I will express the speed of Algorithm C in terms of $d\left(n, x^{k}\right)$. 
Lemma 9.1. Set $f=\lfloor\lg 2 n\rfloor$. At the start of step 3 of Algorithm $C, r \leq x^{k}<$ $r\left(1+2^{-b}\right)$. At the start of step $4, r \leq n$ and $x^{k}<r+2^{f-b}$. At the start of step 5, $n<r+2^{f-b}$.

Proof. By Lemma 6.2 and Lemma 7.1,

$$
r \leq x^{k}<r\left(1+\frac{1}{2^{b+\lceil\lg 4 k\rceil}}\right)^{2 k-1}<r\left(1+\frac{1}{2^{b} 4 k}\right)^{2 k-1}<r\left(1+2^{-b}\right) .
$$

If Algorithm C does not stop in step 3 then $r \leq n<2^{f}$, so $r\left(1+2^{-b}\right)<r+2^{f-b}$. If it also does not stop in step 4 then $n<r\left(1+2^{-b}\right)$.

Lemma 9.2. When Algorithm $C$ stops, it prints the sign of $n-x^{k}$.

Proof. Use each piece of Lemma 9.1. If Algorithm C stops in step 3 then $n<r$; but $r \leq x^{k}$ so $n<x^{k}$. If it stops in step 4 then $r\left(1+2^{-b}\right) \leq n$; but $x^{k}<r\left(1+2^{-b}\right)$ so $x^{k}<n$. If it stops in step 5 then $b \geq f$, so $r \leq n<r+1$ and $r \leq x^{k}<r+1$, so $\left|n-x^{k}\right|<1$; both $n$ and $x^{k}$ are integers, so $n=x^{k}$.

Lemma 9.3. Set $f=\lfloor\lg 2 n\rfloor$ and $g=\max \left\{1, f-d\left(n, x^{k}\right)\right\}$. Algorithm $C$ stops before step 6 if $b \geq g$.

Proof. I prove the contrapositive. If Algorithm C gets to step 6 then $b<f$. By Lemma 9.1, $r \leq n<r+2^{f-b}$ and $r \leq x^{k}<r+2^{f-b}$, so $\left|n-x^{k}\right|<2^{f-b}$. If $n=x^{k}$ then $d\left(n, x^{k}\right)=0<f-b$; if $n \neq x^{k}$ then $d\left(n, x^{k}\right)=\left\lfloor\lg \left|n-x^{k}\right|\right\rfloor \leq \lg \left|n-x^{k}\right|<$ $f-b$. Either way $b<f-d\left(n, x^{k}\right) \leq g$.

Lemma 9.4. Set $f=\lfloor\lg 2 n\rfloor, b_{j}=\min \left\{2^{j}, f\right\}$, and $g=\max \left\{1, f-d\left(n, x^{k}\right)\right\}$. Then Algorithm $C$ takes $M$-time at most $P(k) \sum_{0 \leq j \leq\lceil\lg g\rceil} M\left(b_{j}+\lceil\lg 8 k\rceil\right)$.

Proof. Each iteration of step 2 uses time at most $P(k) M(b+\lceil\lg 8 k\rceil)$, by Lemma 6.3. Notice that $b_{\lceil\lg f\rceil-1}<f$ but $b_{\lceil\lg f\rceil}=f$. So Algorithm $\mathrm{C}$ uses first $b \leftarrow b_{0}$ then $b \leftarrow b_{1}$, and so on through at most $b \leftarrow b_{\lceil\lg f\rceil}$. If $j>\lceil\lg g\rceil$ then $j \geq 1$ and $b_{j-1}=\max \left\{2^{j-1}, f\right\} \geq \max \left\{2^{\lceil\lg g\rceil}, g\right\} \geq g$, so Algorithm C stops before step 6 with $b \leftarrow b_{j-1}$, so it never gets to $b_{j}$.

Lemma 9.5. Set $f=\lfloor\lg 2 n\rfloor$ and $g=\max \left\{1, f-d\left(n, x^{k}\right)\right\}$. Then Algorithm $C$ takes $M$-time less than $P(k)(4 g+\lceil\lg 2 g\rceil\lceil\lg 8 k\rceil) \mu(2 g+\lceil\lg 8 k\rceil)$.

Proof. Set $b_{j}=\min \left\{2^{j}, f\right\}$. If $j \leq\lceil\lg g\rceil$ then $b_{j} \leq 2^{j}<2 g$, so $M\left(b_{j}+\lceil\lg 8 k\rceil\right) \leq$ $\left(2^{j}+\lceil\lg 8 k\rceil\right) \mu(2 g+\lceil\lg 8 k\rceil)$. Now by Lemma 9.4 the $M$-time is at most

$$
\begin{aligned}
P(k) \sum_{0 \leq j \leq\lceil\lg g\rceil} M\left(b_{j}+\lceil\lg 8 k\rceil\right) & \leq P(k) \sum_{0 \leq j \leq\lceil\lg g\rceil}\left(2^{j}+\lceil\lg 8 k\rceil\right) \mu(2 g+\lceil\lg 8 k\rceil) \\
& <P(k)\left(2^{\lceil\lg 2 g\rceil}+\lceil\lg 2 g\rceil\lceil\lg 8 k\rceil\right) \mu(2 g+\lceil\lg 8 k\rceil) \\
& <P(k)(4 g+\lceil\lg 2 g\rceil\lceil\lg 8 k\rceil) \mu(2 g+\lceil\lg 8 k\rceil)
\end{aligned}
$$

as claimed. 
Notes. My use of increasing precision is at the heart of my improvement over [4]. A 50-digit number that starts with 9876 is not an 11th power; the remaining 46 digits are irrelevant. In general, Algorithm $\mathrm{C}$ does not inspect many more bits of $n$ than are necessary to distinguish $n$ from $x^{k}$. The last step dominates the run time.

In Newton's method it is natural to double the precision at each step. But Algorithm $\mathrm{C}$ could use any vaguely geometric progression. In practice I should modify the $b$ sequence to take into account the speed of multiplication and the distribution of $x$ and $n$.

Lemma 6.3 is hopelessly pessimistic about the time needed to compute $x^{k}$ to high accuracy. Since $x$ has very few bits, the first few multiplications use relatively low precision. In fact, the $P(k)$ factor in Lemma 9.4 should disappear as $g$ grows. Similarly, the bound from Lemma 6.2 is somewhat loose. A careful analysis would show that, when $b$ is large, step 2 of Algorithm $C$ can use fewer than $\lceil\lg 8 k\rceil$ guard bits. This is probably not worth the added complexity in practice.

Step 3 of Algorithm C compares a high-precision number, $n$, to a low-precision floating-point number, $r$. The alert reader may have observed that this is a potential bottleneck. The $M$-time in Algorithm $\mathrm{C}$ is essentially the precision of $r$; this may be much less time than it takes to read the digits of $n$. Fortunately, one can check whether $n<r$ in time proportional to the size of $r$, so there is no difficulty. Similar comments apply to step 4 .

\section{HOW TO TEST IF $n$ IS A $k$ TH POWER}

Let $n$ and $k$ be integers larger than 1 . Algorithm $\mathrm{K}$ checks whether $n$ is a $k$ th power. The idea is to compute a floating-point approximation $r$ to $n^{1 / k}$; say $\left|n^{1 / k}-r\right|<1 / 4$. Then, if $r$ is within $1 / 4$ of an integer $x$, check whether $x^{k}=n$.

This algorithm uses a precomputed approximation $y$ to $n^{-1}$. See Lemma 10.2.

Algorithm K. Given integers $n \geq 2$ and $k \geq 2$, and a positive floating-point number $y$, to see if $n$ is a $k$ th power: In advance set $f=\lfloor\lg 2 n\rfloor$ and $b=3+\lceil f / k\rceil$.

1. Compute $r \leftarrow \operatorname{nroot}_{b}(y, k)$.

2. Find an integer $x$ with $|r-x| \leq 5 / 8$.

3. If $x=0$ or $|r-x| \geq 1 / 4$, print 0 and stop.

4. Compute the sign of $n-x^{k}$ with Algorithm C.

5. If $n=x^{k}$, print $x$ and stop.

6. Print 0 .

Lemma 10.1. $\sqrt{1+t} /(1-t) \leq 1+2 t$ and $\sqrt{1-t} /(1+t) \geq 1-2 t$ if $0 \leq t \leq 1 / 10$.

Lemma 10.2. $\left|r-n^{1 / k}\right|<1 / 4$ in Algorithm $K$ if $y\left(1-2^{-b}\right)<n^{-1}<y\left(1+2^{-b}\right)$.

Proof. Write $\delta=2^{-b}$. By hypothesis, $y(1-\delta)<n^{-1}<y(1+\delta)$, so $y^{-1 / k}(1+\delta)^{-1 / k}<n^{1 / k}<y^{-1 / k}(1-\delta)^{-1 / k}$. By Lemma 8.7, $r(1-\delta)<y^{-1 / k}$ $<r(1+\delta)$. Note that $\delta \leq 1 / 16<1 / 10$. Apply Lemma 10.1 twice: first

$$
\begin{aligned}
r & <\frac{y^{-1 / k}}{1-\delta}<n^{1 / k} \frac{(1+\delta)^{1 / k}}{1-\delta} \leq n^{1 / k} \frac{(1+\delta)^{1 / 2}}{1-\delta}=n^{1 / k}+n^{1 / k}\left(\frac{(1+\delta)^{1 / 2}}{1-\delta}-1\right) \\
& \leq n^{1 / k}+n^{1 / k} 2 \delta<n^{1 / k}+2^{f / k} 2 \delta=n^{1 / k}+2^{f / k} 2^{-2-\lceil f / k\rceil} \leq n^{1 / k}+\frac{1}{4}
\end{aligned}
$$


second

$$
\begin{aligned}
r & >\frac{y^{-1 / k}}{1+\delta}>n^{1 / k} \frac{(1-\delta)^{1 / k}}{1+\delta} \geq n^{1 / k} \frac{(1-\delta)^{1 / 2}}{1+\delta}=n^{1 / k}+n^{1 / k}\left(\frac{(1-\delta)^{1 / 2}}{1+\delta}-1\right) \\
& \geq n^{1 / k}-n^{1 / k} 2 \delta>n^{1 / k}-2^{f / k} 2 \delta=n^{1 / k}-2^{f / k} 2^{-2-\lceil f / k\rceil} \geq n^{1 / k}-\frac{1}{4} .
\end{aligned}
$$

Hence $\left|r-n^{1 / k}\right|<1 / 4$.

Lemma 10.3. Set $f=\lfloor\lg 2 n\rfloor$ and $b=3+\lceil f / k\rceil$. Assume that $y\left(1-2^{-b}\right)<$ $n^{-1}<y\left(1+2^{-b}\right)$. If $n$ is a $k$ th power, Algorithm K prints $n^{1 / k}$. If $n$ is not a $k$ th power, Algorithm K prints 0.

Proof. Algorithm K finds an integer $x$ with $|r-x| \leq 5 / 8$. By Lemma 10.2, $\left|r-n^{1 / k}\right|<1 / 4$, so $\left|x-n^{1 / k}\right|<1 / 4+5 / 8<1$. If $n$ is a $k$ th power then $x$ and $n^{1 / k}$ are both integers so $x=n^{1 / k}$. Then $|r-x|=\left|r-n^{1 / k}\right|<1 / 4$ and $x>0$, so Algorithm K does not stop in step 3 ; in step 5 it prints $x$.

On the other hand, if $n$ is not a $k$ th power, then certainly $n \neq x^{k}$, so Algorithm $\mathrm{K}$ does not stop in step 5 . So it prints 0 .

Let $t$ be a real number such that $t-1 / 2$ is not an integer. Write round $t$ for the nearest integer to $t$ : the unique integer $i$ with $|i-t|<1 / 2$.

Lemma 10.4. Set $f=\lfloor\lg 2 n\rfloor, g=\max \left\{1, f-d\left(n,\left(\operatorname{round} n^{1 / k}\right)^{k}\right)\right\}$, and $b=$ $3+\lceil f / k\rceil$. Assume that $y\left(1-2^{-b}\right)<n^{-1}<y\left(1+2^{-b}\right)$. Then Algorithm $K$ uses $M$-time less than

$$
\begin{aligned}
(4 g+\lceil\lg 2 g\rceil\lceil\lg 8 k\rceil) P(k) \mu(2 g+\lceil\lg 8 k\rceil)+\lceil\lg 4 k\rceil(P(k)+1) M(\lceil\lg (66(2 k+1))\rceil) \\
+(2\lfloor f / k\rfloor+\lfloor\lg f\rfloor\lceil 8+\lg k\rceil)(P(k+1)+1) \mu(\lceil f / k\rceil+9) .
\end{aligned}
$$

Proof. Define $T=\lceil\lg 4 k\rceil(P(k)+1) M(\lceil\lg (66(2 k+1))\rceil)$. Note that $b-\lceil\lg 2 k\rceil=$ $\lceil f / k\rceil-\lceil\lg k\rceil+2 \leq\lceil f / 2\rceil+1 \leq f$ since $f \geq 2$.

Algorithm K first computes $\operatorname{nroot}_{b}(y, k)$. If $b \leq\lceil\lg 8 k\rceil$ then, by Lemma 8.1, this uses $M$-time at most $T$. If $b>\lceil\lg 8 k\rceil$ then, by Lemma 8.4, it uses $M$-time at most

$$
\begin{aligned}
T+ & (P(k+1)+1)(2(b-\lceil\lg 8 k\rceil)+\lceil 8+\lg k\rceil\lceil\lg (b-\lceil\lg 2 k\rceil)-1\rceil) \mu(b+6) \\
& \leq T+(2(\lceil f / k\rceil-1)+\lceil 8+\lg k\rceil\lceil\lg f-1\rceil)(P(k+1)+1) \mu(\lceil f / k\rceil+9) \\
& \leq T+(2\lfloor f / k\rfloor+\lceil 8+\lg k\rceil\lfloor\lg f\rfloor)(P(k+1)+1) \mu(\lceil f / k\rceil+9) .
\end{aligned}
$$

After computing $r=\operatorname{nroot}_{b}(y, k)$, Algorithm $\mathrm{K}$ finds an integer $x$. It may invoke Algorithm C; if it does, then $|r-x|<1 / 4$, and $\left|r-n^{1 / k}\right|<1 / 4$ by Lemma 10.2, so $\left|x-n^{1 / k}\right|<1 / 2$, so $x=$ round $n^{1 / k}$. Finally, by Lemma 9.5, Algorithm C uses $M$-time at most $P(k)(4 g+\lceil\lg 2 g\rceil\lceil\lg 8 k\rceil) \mu(2 g+\lceil\lg 8 k\rceil)$.

\section{How tO TEST IF $n$ IS A PERFECT POWER}

To see whether $n$ is a perfect power, I run through the primes $p \leq \lg n$; I check for each $p$ whether $n$ is a $p$ th power. For a time analysis see the next section. 
Algorithm $X$. Given an integer $n \geq 2$, to decompose $n$ as a perfect power if possible: In advance set $f=\lfloor\lg 2 n\rfloor$.

1. Compute $y \leftarrow \operatorname{nroot}_{3+\lceil f / 2\rceil}(n, 1)$.

2. For each prime number $p<f$ :

3. $\quad$ Apply Algorithm $\mathrm{K}$ to $(n, p, y)$; let $x$ be the result.

4. $\quad$ If $x>0$, print $(x, p)$ and stop.

5. Print $(n, 1)$.

Lemma 11.1. $y\left(1-2^{-3-\lceil f / p\rceil}\right)<n<y\left(1+2^{-3-\lceil f / p\rceil}\right)$ in Algorithm $X$.

Proof. $y\left(1-2^{-3-\lceil f / 2\rceil}\right)<n<y\left(1+2^{-3-\lceil f / 2\rceil}\right)$ by Lemma 8.7 ; and $p \geq 2$.

Lemma 11.2. If $n$ is a perfect power, Algorithm $X$ prints a prime number $p$ and a positive integer $x$ such that $x^{p}=n$. If $n$ is not a perfect power, Algorithm $X$ prints $(n, 1)$.

Proof. By Lemma 11.1, $y\left(1-2^{-3-\lceil f / p\rceil}\right)<n<y\left(1+2^{-3-\lceil f / p\rceil}\right)$. If Algorithm X stops in step 4 then, by Lemma 10.3, $x^{p}=n$.

Conversely, if $n$ is a perfect power then $n$ is a $p$ th power for some prime $p \leq$ $\lg n<f$. By Lemma 10.3, Algorithm X stops in step 4 .

Notes. The result of [4] is a perfect-power classification algorithm that runs in time $\log ^{3} n$; on average, under reasonable assumptions, it runs in time $\log ^{2} n / \log ^{2} \log n$.

The run time of Algorithm $\mathrm{X}$ is much better: it is essentially linear in $\log n$, given fast multiplication. The proof uses transcendental number theory. For further discussion see section 12 .

Algorithm $\mathrm{X}$ is not new. It is stated in, e.g., [17, section 2.4]. But God is in the details: without good methods for computing $n^{1 / k}$ and for checking whether $x^{k}=n$, Algorithm $\mathrm{X}$ is not so attractive. The authors of [17] go on to say that one can "save time" by adding a battery of tests to Algorithm X. Variants of Algorithm $\mathrm{X}$ are also dismissed in [11, page 38] ("This is clearly quite inefficient") and [4]. Observe that, by putting enough work into the subroutines, I have made Algorithm $\mathrm{X}$ quite fast - so fast, in fact, that typical modifications will slow it down.

I use the Sieve of Eratosthenes to enumerate the primes $p<f$. See [27] for faster methods. Note that the best order of operations in Algorithm X depends on the distribution of inputs; for example, if the input source is very likely to produce 37 th powers, then $p=37$ should be done first.

\section{IntRoduCtion to $F(n)$}

Define

$$
F(n)=\sum_{2 \leq p \leq \lg n}(\lg p) \max \left\{1, \lg n-d\left(n,\left(\operatorname{round} n^{1 / p}\right)^{p}\right)\right\}
$$

for $n \geq 2$. Here $p$ is prime, and round $t$ means an integer within $1 / 2$ of $t$.

$F(n)$ has about $\lg n / \log \lg n$ terms, labelled by prime exponents $p \leq \lg n$. The $p$ term reflects the difficulty of determining that $n$ is not a $p$ th power. In each term, the main factor $\max \left\{1, \lg n-d\left(n,\left(\operatorname{round} n^{1 / p}\right)^{p}\right)\right\}$ says how many bits of $n$ agree with a nearby $p$ th power. If $n$ is very close to a $p$ th power then this factor is close to $\lg n$. The minor factor $\lg p$ represents the effort spent computing $p$ th powers. 
$F(n)$ is the subject of Part IV and Part V. Part IV gives lower and upper bounds for $F$, and shows that the normal and average behaviors of $F$ are comparable to the lower bound.

Part V shows that $F(n)$ is bounded by $(\lg n)^{1+\epsilon(n)}$ for a certain function $\epsilon \in o(1)$. The approach is through the following application of transcendental number theory: there cannot be many perfect powers in a short interval. This means that there are not many perfect powers close to $n$, so not many of the main factors in $F(n)$ are near $\lg n$. Note that the exponent $1+\epsilon(n)$, albeit theoretically satisfying, is ridiculously large for any reasonable value of $n$.

Lemma 12.1. Set $f=\lfloor\lg 2 n\rfloor$. For $n \geq 2$, Algorithm $X$ takes $M$-time at most $\left(8 F(n)+6 f\lfloor\lg 16 f\rfloor^{3}\right) \mu(2 f+\lfloor\lg 128 f\rfloor)$.

The reader may verify that Algorithm X does not use much non- $M$-time. Hence Algorithm X takes time essentially linear in $F(n)+\log n$, provided that it uses a fast multiplication algorithm. Since $F(n)$ is essentially linear in $\log n$, the run time of Algorithm X is essentially linear in $\log n$.

Proof. Write $c=\lfloor\lg f\rfloor$. Note that $\lceil\lg f\rceil \leq c+1$. Note also that $\sum_{2 \leq p<f} 1 / p<c$.

Step 1 computes $y=\operatorname{nroot}_{3+\lceil f / 2\rceil}(n, 1)$. By Lemma 8.4 this takes $M$-time at most $2 M(8)+2(f+8\lfloor\lg 4 f\rfloor) \mu(\lceil f / 2\rceil+9)$.

Each iteration of step 3 invokes Algorithm $\mathrm{K}$ for a prime number $p<f$. Write $g=g_{p}=\max \left\{1, f-d\left(n,\left(\operatorname{round} n^{1 / p}\right)^{p}\right)\right\}$. By Lemma 11.1, Lemma 10.4, and Lemma 6.1, Algorithm K takes $M$-time less than

$$
\begin{aligned}
(4 g+ & \lceil\lg 2 g\rceil\lceil\lg 8 p\rceil) P(p) \mu(2 g+\lceil\lg 8 p\rceil)+\lceil\lg 4 p\rceil(P(p)+1) M(\lceil\lg (66(2 p+1))\rceil) \\
& +(2\lfloor f / p\rfloor+\lfloor\lg f\rfloor\lceil 8+\lg p\rceil)(P(p+1)+1) \mu(\lceil f / p\rceil+9) \\
< & 8 g\lfloor\lg p\rfloor \mu(2 f+c+4)+4\lfloor f / p\rfloor(c+1) \mu(f+9) \\
& +2(c+1)((c+2)(c+4)+(c+3)(c+9)+c(c+9)) \mu(2 f+c+7) \\
< & \left(8 g \lg p+4(c+1) f / p+6(c+1)\left(c^{2}+9 c+12\right)\right) \mu(2 f+c+7) .
\end{aligned}
$$

The total $M$-time is then less than $\mu(2 f+c+7)$ times

$$
\begin{aligned}
2 f+ & 16(c+3)+\sum_{2 \leq p \leq f-1}\left(8 g_{p} \lg p+4(c+1) f / p+6(c+1)\left(c^{2}+9 c+12\right)\right) \\
& <2 f+16(c+3)+8 F(n)+4(c+1) f c+6 f(c+1)\left(c^{2}+9 c+12\right) \\
& \leq 8 F(n)+2 f\left(1+4(c+3)+2 c(c+1)+3(c+1)\left(c^{2}+9 c+12\right)\right) \\
& <8 F(n)+6 f(c+4)^{3}
\end{aligned}
$$

as claimed.

Notes. $F(n)$ is generally not the dominant term in Lemma 12.1; see Part IV. The reader may be tempted to chop one or more $\lg f$ factors out of the other term by, for example, using known bounds on the functions $\vartheta, \vartheta_{2}, \ell$ defined in section 14 , or by assuming that $\mu(b)$ grows at least as quickly as $\lg b$. However, this is a pointless exercise: several variants of Algorithm X appear in Part VI, and it is easier to achieve any desired run-time goal with one of those variants than with the original algorithm. 


\section{Proof of Theorem 1}

In this section I combine all my results to prove Theorem 1: there is a perfectpower classification algorithm that uses time at most $(\lg n)^{1+o(1)}$ for $n \rightarrow \infty$.

Let $T(n)$ be an upper bound on the time taken by Algorithm $\mathrm{X}$ for $n \geq 2$. As discussed in section 12 , one may take $T(n) \in(\lg n)^{1+o(1)}$ for $n>2$, given fast multiplication.

Define $U(n)=\max \{T(m) / \lg m: m \leq n\} \lg n$ for $n \geq 2$.

Now $T(m) / \lg m \in(\lg m)^{o(1)}$ for $m>2$, so $U(n) / \lg n \in(\lg n)^{o(1)}$ for $n>2$ by Lemma 3.1. Hence $U(n) \in(\lg n)^{1+o(1)}$.

To finish the proof I exhibit a perfect-power classification algorithm, Algorithm $\mathrm{PPC}$, and prove that it runs in time $2 U(n)$.

Algorithm PPC. Given $n \geq 2$, to print $(x, k)$ such that (1) $x^{k}=n$ and (2) $x$ is not a perfect power:

1. Apply Algorithm $\mathrm{X}$ to $n$; let $(x, p)$ be the result.

2. If $(x, p)=(n, 1)$, print $(n, 1)$ and stop.

3. (Note that $2 \leq x<n$.) Apply Algorithm PPC to $x$; let $(c, k)$ be the result.

4. Print $(c, k p)$.

Lemma 13.1. If $n=x^{p}$ then $p U(x) \leq U(n)$.

Proof. $U(n) / \lg n$ is a nondecreasing function of $n$, so $p U(x) / \lg x \leq p U(n) / \lg n=$ $p U(n) / p \lg x=U(n) / \lg x$.

Lemma 13.2. Algorithm PPC spends time at most $2 U(n)$ plus housekeeping.

Proof. Step 1 takes time at most $T(n)$. If $n$ is a perfect power then Algorithm PPC calls itself recursively; by induction this takes time at most $2 U(x)$. The total time is at most $T(n)+2 U(x) \leq U(n)+p U(x) \leq 2 U(n)$ by Lemma 13.1.

\section{PART IV. ANALYTIC METHODS}

\section{Intuition ABout $F(n)$}

In this section, I give some motivation for the facts about $F(n)$ proved in the next section. The theme here is that $F(n)$ is roughly $\lg n \lg \lg n$.

$F(n)$ is a sum over primes $p$. I will analyze it in terms of the following three simpler sums: $\vartheta(t)=\sum_{2 \leq p \leq t} \log p \approx t ; \vartheta_{2}(t)=\sum_{2 \leq p \leq t} \log ^{2} p \approx t \log t-t ; \ell(t)=$ $\sum_{2 \leq p \leq t}(\log p) / p \approx \log t$.

Fix $p$, and define $u$ as follows: $n$ is $u p n^{1-1 / p}$ away from the nearest $p$ th power. Then $u$ is, intuitively, a random number between 0 and $1 / 2$. Indeed, if $n$ is randomly selected from the interval $\left[x^{p},(x+1)^{p}\right]$, then its distance to the nearest endpoint ranges uniformly from 0 to $\left((x+1)^{p}-x^{p}\right) / 2 \approx(1 / 2) p x^{p-1} \approx(1 / 2) p n^{1-1 / p}$.

These approximations break down when $x \approx n^{1 / p}$ is smaller than $p$, so assume for the moment that $p$ is at most $\lg n / \lg \lg n$.

The number of bits I need to distinguish $n$ from the nearest $p$ th power is about $\lg n-\lg u p n^{1-1 / p}=(1 / p) \lg n-\lg p-\lg u$. If in fact $u$ were uniformly distributed between 0 and $1 / 2$, then the average value of $\lg u$ would be $2 \int_{0}^{1 / 2} \lg u d u=-1-$ $1 / \log 2$. So I estimate the number of bits, on average, as $(1 / p) \lg n-\lg p+1+1 / \log 2$. Note that this is positive, since $p<n^{1 / p}$. 
As $p$ grows past $\lg n / \lg \lg n$, on the other hand, the $p$ th powers become so widely spaced that I usually need only a single bit of $n$.

Now consider $F(n) . F(n)$ compares $n$ with the $p$ th power of the integer closest to $n^{1 / p}$; this is usually the nearest $p$ th power to $n$. So I estimate that, on average,

$$
\begin{aligned}
F(n) & \approx \sum_{p \leq \lg n / \lg \lg n}\left(\frac{\lg p}{p} \lg n-\lg ^{2} p+\left(1+\frac{1}{\log 2}\right) \lg p\right)+\sum_{\lg n / \lg \lg n<p \leq \lg n} \lg p \\
& \approx \frac{1}{\log ^{2} 2}\left(\ell\left(\frac{\lg n}{\lg \lg n}\right) \log n-\vartheta_{2}\left(\frac{\lg n}{\lg \lg n}\right)+\vartheta\left(\frac{\lg n}{\lg \lg n}\right) \log 2 e\right)+\frac{\vartheta(\lg n)}{\log 2} \\
& \approx \lg n \lg \lg n-\lg n \lg \lg \lg n+\frac{\lg n}{\lg \lg n} \frac{\log \lg \lg n+\log 2+2}{\log ^{2} 2} .
\end{aligned}
$$

What makes $F(n)$ difficult to analyze is that $u$ is occasionally very close to 0 . Then $-\lg u$ is much larger than its usual value. If this happens for a few primes $p$-as it does, for example, when $n \approx 32768$ - then $F(n)$ will be noticeably larger than expected. I will get a lower bound on $F(n)$ by changing $u$ to 1 , but I cannot get an upper bound in any analogous way.

Notes. See [28] for bounds on $\vartheta$. See [3, section 2.7] for a general approach to obtaining bounds on functions such as $\vartheta, \vartheta_{2}$, and $\ell$.

\section{Analysis of $F(n)$}

Lemma 15.1 gives a lower bound for $F(n)$, roughly $\lg \lg n-\lg \lg \lg n-1 / \log 2$ times $\lg n$. Lemma 15.2 gives a weak (quadratic) upper bound for $F(n)$. Lemma 15.4 (in light of Lemma 15.3) gives a much better upper bound for the normal behavior of $F(n)$, roughly $(1+2 / \log 2)(\lg n \lg \lg n)$. Combining Lemmas 15.2, 15.3, and 15.4 produces about the same bound for the average behavior of $F(n)$. A more careful analysis, omitted to save space, shows that the average for $2^{f-1} \leq n<2^{f}$ is at most $(2 / \log 2) f \ell(f-1)+(12 / \log 2) \vartheta(f-1)$, roughly $2 \lg n \lg \lg n$, if $f \geq 10$.

These results translate directly into facts about the run time of my perfect-power decomposition algorithm, Algorithm X. See Lemma 12.1.

Lemma 15.1. If $n \geq 4$ then

$$
F(n)>\frac{1}{\log 2} \ell\left(\frac{\lg n}{\lg \lg n}\right) \lg n-\frac{1}{\log ^{2} 2} \vartheta_{2}\left(\frac{\lg n}{\lg \lg n}\right) .
$$

Proof. Note that $\lg \lg n \geq 1$. Fix $p \leq \lg n / \lg \lg n$, so that $p \leq \lg n \leq n^{1 / p}$. Set $x=\operatorname{round} n^{1 / p}$.

If $n \geq x^{p}$ then

$$
\begin{aligned}
n-x^{p} & =\left(n^{1 / p}-x\right)\left(n^{1-1 / p}+x n^{1-2 / p}+\cdots+x^{p-1}\right) \\
& \leq \frac{1}{2}\left(n^{1-1 / p}+n^{1-1 / p}+\cdots+n^{1-1 / p}\right)=\frac{p}{2} n^{1-1 / p} .
\end{aligned}
$$

If $n<x^{p}$ then

$$
\begin{aligned}
x^{p}-n & =\left(x-n^{1 / p}\right)\left(x^{p-1}+x^{p-2} n^{1 / p}+\cdots+n^{1-1 / p}\right) \leq \frac{p}{2} x^{p-1} \\
& <\frac{p}{2}\left(n^{1 / p}+\frac{1}{2}\right)^{p-1}<\frac{p}{2} n^{1-1 / p}\left(1+\frac{1}{2 p}\right)^{p}<\frac{p}{2} n^{1-1 / p} e^{1 / 2} .
\end{aligned}
$$


Either way $\left|n-x^{p}\right|<p n^{1-1 / p}$, so $d\left(n, x^{p}\right)<(1-1 / p) \lg n+\lg p$. Hence

$$
F(n)>\sum_{p \leq \lg n / \lg \lg n}(\lg p)\left(\frac{1}{p} \lg n-\lg p\right)=\ell\left(\frac{\lg n}{\lg \lg n}\right) \frac{\lg n}{\log 2}-\vartheta_{2}\left(\frac{\lg n}{\lg \lg n}\right) \frac{1}{\log ^{2} 2}
$$

as claimed.

Lemma 15.2. $F(n) \leq \vartheta(\lg n) \lg n / \log 2$.

Proof. $\lg n-d\left(n,\left(\operatorname{round} n^{1 / p}\right)^{p}\right) \leq \lg n$, and $\sum_{2 \leq p \leq \lg n} \lg p=\vartheta(\lg n) / \log 2$.

For the next two lemmas I say that $n$ is exceptional for $p$ if it is within $n^{1-1 / p} / \lg ^{2} n$ of a $p$ th power. I say that $n$ is exceptional if it is exceptional for some prime $p \leq \lg n$.

Lemma 15.3. There are at most $2^{3+f} /(f-1)+2^{2+f / 2}(f-1)$ exceptional integers $n$ in the interval $2^{f-1} \leq n<2^{f}$.

Proof. Fix $p$. Set $T=2^{f-f / p} /(f-1)^{2}$; if $n$ is exceptional for $p$ then $n$ differs from some $p$ th power by less than $T$. Write $I=\left[2^{f-1}, 2^{f}-1\right]$.

Let $S$ be the set of integers $x$ between $\left\lceil 2^{(f-1) / p}-1\right\rceil$ and $\left\lfloor 2^{f / p}+1\right\rfloor$ inclusive. Say $\left|n-y^{p}\right|<T$ with $n \in I$. Then there is an $x \in S$ such that $\left|n-x^{p}\right|<T$ : if $y \in S$, take $x=y$; if $y>\left\lfloor 2^{f / p}+1\right\rfloor$, take $x=\left\lfloor 2^{f / p}+1\right\rfloor$; if $y<\left\lceil 2^{(f-1) / p}-1\right\rceil$, take $x=\left[2^{(f-1) / p}-1\right\rceil$.

There are $\left\lfloor 2^{f / p}\right\rfloor-\left\lceil 2^{(f-1) / p}\right\rceil+3<2^{2+f / p}$ elements $x \in S$. Each $x$ produces at most $2 T+1$ integers exceptional for $p$. Thus there are at most $2^{2+f / p}(2 T+1) \leq$ $2^{3+f} /(f-1)^{2}+2^{2+f / 2}$ integers in $I$ exceptional for $p$.

There are at most $f-1$ primes $p$, so there are at most $2^{3+f} /(f-1)+2^{2+f / 2}(f-1)$ exceptional integers in $I$.

Lemma 15.4. $F(n)<\ell(\lg n) \lg n / \log 2+(2 \lg \lg n+1) \vartheta(\lg n) / \log 2$ if $n$ is not exceptional and $n \geq 4$.

Proof. By hypothesis $d\left(n, x^{p}\right)>\lg n^{1-1 / p}-2 \lg \lg n-1$ for any $x$ and any $p \leq \lg n$. So $\lg n-d\left(n, x^{p}\right)<(1 / p) \lg n+2 \lg \lg n+1$. Thus

$$
\begin{aligned}
F(n) & <\sum_{p \leq \lg n}(\lg p)((1 / p) \lg n+2 \lg \lg n+1) \\
& =\sum_{p \leq \lg n}\left(\frac{\lg p}{p} \lg n+(2 \lg \lg n+1) \lg p\right)=\frac{\ell(\lg n)}{\log 2} \lg n+\frac{2 \lg \lg n+1}{\log 2} \vartheta(\lg n) ;
\end{aligned}
$$

note that the sum is nonempty since $n \geq 4$.

Notes. $F(n)$ usually behaves like $\lg n \lg \lg n$, but it behaves more like $2 \lg n \lg \lg n$ when $n$ is a power of 2 with a sufficiently smooth exponent. Is $F(n) / \lg n \lg \lg n$ unbounded?

\section{PART V. TRANSCENDENTAL METHODS \\ 16. Multiplicative DePendence}

I call $x_{0}, \ldots, x_{n}$ multiplicatively dependent if there are integers $a_{0}, \ldots, a_{n}$, not all zero, with $x_{0}^{a_{0}} \cdots x_{n}^{a_{n}}=1$. 
The following lemma, quoted without proof, is a special case of a theorem of Loxton and van der Poorten.

Lemma 16.1. Let $x_{0}, \ldots, x_{n}$ be multiplicatively dependent positive integers with $x_{j} \geq 3$. Then there are integers $a_{0}, \ldots, a_{n}$, not all zero, with $x_{0}^{a_{0}} \cdots x_{n}^{a_{n}}=1$, and $\left|a_{j}\right|<3 n^{n}\left(\log x_{0}\right) \cdots\left(\log x_{n}\right)$.

Notes. Lemma 16.1 follows from [21, Theorem $5(\mathrm{~A})]$, with $D=1, w(\mathbf{Q})=2$, and $\lambda(1)=\log 2$; note that $1<\log x_{j}$ and $2\left(n ! /(\log 2)^{n}\right)<3 n^{n}$.

\section{LiNEAR FORMS IN LOGARITHMS}

The height of a nonzero rational number $\alpha$ is $H(\alpha)=\max \{|i|,|j|\}$, if $\alpha=i / j$ in lowest terms. The height of 0 is 0 .

The following lemma, quoted without proof, is a special case of a theorem of Loxton.

Lemma 17.1. Fix $c \geq 1, n \geq 1$. Let $\alpha_{1}, \ldots, \alpha_{n}$ be multiplicatively independent positive rational numbers. Let

$$
\left(\begin{array}{cccc}
\beta_{11} & \beta_{12} & \cdots & \beta_{1 n} \\
\beta_{21} & \beta_{22} & \cdots & \beta_{2 n} \\
\vdots & \vdots & \ddots & \vdots \\
\beta_{c 1} & \beta_{c 2} & \cdots & \beta_{c n}
\end{array}\right)
$$

be a rank-c matrix of rational numbers. Fix $A_{j} \geq 4$ and $B \geq 4$ such that $H\left(\alpha_{j}\right) \leq$ $A_{j}$ and $H\left(\beta_{i j}\right) \leq B$. Write $\Omega=\left(\log A_{1}\right) \cdots\left(\log A_{n}\right)$. Write

$$
\left(\begin{array}{c}
\Lambda_{1} \\
\Lambda_{2} \\
\vdots \\
\Lambda_{c}
\end{array}\right)=\left(\begin{array}{cccc}
\beta_{11} & \beta_{12} & \cdots & \beta_{1 n} \\
\beta_{21} & \beta_{22} & \cdots & \beta_{2 n} \\
\vdots & \vdots & \ddots & \vdots \\
\beta_{c 1} & \beta_{c 2} & \cdots & \beta_{c n}
\end{array}\right)\left(\begin{array}{c}
\log \alpha_{1} \\
\log \alpha_{2} \\
\vdots \\
\log \alpha_{n}
\end{array}\right) .
$$

Then, for some $i,\left|\Lambda_{i}\right|>\exp \left(-(16 n)^{200 n}(\Omega \log \Omega)^{1 / c} \log B \Omega\right)$.

Notes. A central theorem of Baker [5] states that a single nonzero linear form in logarithms cannot be exceedingly close to 0 , or in fact to any algebraic number. Loxton's theorem [20, Theorem 4] generalizes Baker's theorem to handle several independent linear forms in the same set of logarithms. Lemma 17.1 follows from [20, Theorem 4] with $d=1$.

The constants 16 and 200 here can easily be reduced.

\section{More InEQUalities}

Lemma 18.1. If $x_{1}{ }^{k_{1}} \in[L, U]$ and $x_{2}{ }^{k_{2}} \in[L, U]$ then $\left|k_{1} \log x_{1}-k_{2} \log x_{2}\right| \leq$ $\log (U / L)$.

Lemma 18.2. For $u \geq 1000$ set $T=(1 / 10) \sqrt{u / \log 2.56 u}$. Then $T>1,4 T+2<\sqrt{u}$, $200 T \log 16 T<u / T=10 \sqrt{u \log 2.56 u}, 6 T<e^{u}$, and $T(7+\lg T+u / \log 2-\lg \log 2)<u^{3}$.

Lemma 18.3. If $v \geq 1$ and $t \geq 5$ then $\log \left(t^{v}+t^{v-1}\right)<-2 v \log \log ((t+1) /(t-1))$.

Lemma 18.4. If $\log \log 16 \leq t \leq 1600$ then $t-\log \log 2<40 \sqrt{t \log t}$.

Lemma 18.5. For $n \geq \exp \exp 1000$ write $t=\log \log n$ and $u=\log \log 2 n$. Then $6 u^{3} \exp (30 \sqrt{u \log 2.56 u})<\exp (40 \sqrt{t \log t})$. 


\section{Powers in SHORT InTERVALS}

In this section, I combine the lemmas stated in the previous three sections to show that a short interval $[L, U]$ cannot contain many perfect powers. (These results are due primarily to John Loxton. See the notes at the end of this section.)

What I really count is the number of exponents $k$ such that there is a $k$ th power in $[L, U]$. Lemma 19.2 is my workhorse: it says that there can be very few "large" exponents $k$. Lemma 19.4 gives an upper bound for the number of prime exponents $k$. Corollary 19.5, included here for historical reasons, counts the number of perfect powers in $[L, U]$ when $U=L+\sqrt{L}$.

Lemma 19.1. The matrix

$$
\left(\begin{array}{cccc}
k_{1}+t a_{1} & t a_{2} & \cdots & t a_{m} \\
t a_{1} & k_{2}+t a_{2} & \cdots & t a_{m} \\
\vdots & \vdots & \ddots & \vdots \\
t a_{1} & t a_{2} & \cdots & k_{m}+t a_{m}
\end{array}\right)
$$

has determinant $k_{1} \cdots k_{m}\left(1+t a_{1} / k_{1}+t a_{2} / k_{2}+\cdots+t a_{m} / k_{m}\right)$ for $k_{1}, \ldots, k_{m} \neq 0$.

Proof. Subtract the first row from all succeeding rows; divide column $i$ by $k_{i}$; add each column to the first column. The resulting matrix is upper triangular, with $1+t a_{1} / k_{1}+t a_{2} / k_{2}+\cdots+t a_{m} / k_{m}$ in the top left and 1 elsewhere on the diagonal.

Lemma 19.2. Fix an interval $[L, U]$ with $1<U / e<L<U$. Fix an integer $C \geq 1$. Fix $K \geq 4$ such that

$$
K \geq(16 C)^{200 C} \frac{\log U}{-\log \log (U / L)}(\log U)^{1 / C}((C+1) \log \log U)^{2}
$$

and

$$
K \geq(16 C)^{200 C} \frac{\log U}{-\log \log (U / L)}\left(\log 6 C^{C}+(2 C+1) \log \log U\right)^{2} .
$$

Let $S$ be a set of integer pairs $(x, k)$ with $x^{k} \in[L, U], x \geq 4, k \geq K$. Assume that $k$ and $k^{\prime}$ are coprime whenever $(x, k)$ and $\left(x^{\prime}, k^{\prime}\right)$ are two distinct pairs in $S$. Then $\# S \leq C+\lg C !+C \lg \lg U$.

Note that $\log U>1$ and $-\log \log (U / L)>0$.

Proof. Step 1. Let $\left(x_{1}, k_{1}\right), \ldots,\left(x_{m}, k_{m}\right) \in S$ be multiplicatively independent; here I say that $\left(x_{1}, k_{1}\right), \ldots,\left(x_{m}, k_{m}\right)$ are multiplicatively dependent if $x_{1}, \ldots, x_{m}$ are multiplicatively dependent. I claim that $m \leq C$.

Suppose not: suppose there are $m \geq C+1$ multiplicatively independent pairs $\left(x_{1}, k_{1}\right), \ldots,\left(x_{m}, k_{m}\right) \in S$. Then, in particular, $x_{1}, \ldots, x_{C+1}$ are multiplicatively independent. Put $B=\max \left\{k_{j}: 1 \leq j \leq C+1\right\}$ and $\Omega=\prod_{1 \leq i \leq C+1} \log x_{j}$. Notice that

$$
B \Omega \leq \prod_{1 \leq i \leq C+1} k_{j} \log x_{j} \leq(\log U)^{C+1}
$$


Now

$$
\left(\begin{array}{c}
k_{1} \log x_{1}-k_{C+1} \log x_{C+1} \\
k_{2} \log x_{2}-k_{C+1} \log x_{C+1} \\
\vdots \\
k_{C} \log x_{C}-k_{C+1} \log x_{C+1}
\end{array}\right)=\left(\begin{array}{ccccc}
k_{1} & 0 & \cdots & 0 & -k_{C+1} \\
0 & k_{2} & \cdots & 0 & -k_{C+1} \\
\vdots & \vdots & \ddots & \vdots & \vdots \\
0 & 0 & \cdots & k_{C} & -k_{C+1}
\end{array}\right)\left(\begin{array}{c}
\log x_{1} \\
\vdots \\
\log x_{C+1}
\end{array}\right) .
$$

The conditions of Lemma 17.1 are met: each $x_{j}$ is a positive integer; the matrix has rank $C ; x_{j} \geq 4$ and $B \geq K \geq 4 ; H\left(x_{j}\right)=x_{j} ; H(0)=0 \leq B$; and $H\left(-k_{j}\right)=$ $H\left(k_{j}\right)=k_{j} \leq B$. Hence, for some $i$,

$$
\left|k_{i} \log x_{i}-k_{C+1} \log x_{C+1}\right|>\exp \left(-(16 C)^{200 C}(\Omega \log \Omega)^{1 / C} \log B \Omega\right) .
$$

Apply Lemma 18.1 and take logarithms:

$$
\log \log (U / L)>-(16 C)^{200 C}(\Omega \log \Omega)^{1 / C} \log B \Omega .
$$

Hence

$$
\begin{aligned}
K(-\log \log (U / L)) & <(16 C)^{200 C} K \Omega^{1 / C}(\log \Omega)^{1 / C} \log B \Omega \\
& <(16 C)^{200 C} K^{1+1 / C} \Omega^{1 / C}(\log B \Omega)^{1 / C} \log B \Omega \\
& =(16 C)^{200 C}\left(K^{C+1} \prod_{1 \leq i \leq C+1} \log x_{i}\right)^{1 / C}(\log B \Omega)^{1+1 / C} \\
& \leq(16 C)^{200 C}\left(\prod_{1 \leq i \leq C+1} k_{i} \log x_{i}\right)^{1 / C}(\log B \Omega)^{2} \\
& \leq(16 C)^{200 C}\left(\prod_{1 \leq i \leq C+1} \log U\right)^{1 / C}((C+1) \log \log U)^{2} \\
& =(16 C)^{200 C}(\log U)^{1+1 / C}((C+1) \log \log U)^{2} \\
& \leq K(-\log \log (U / L))
\end{aligned}
$$

Contradiction.

Step 2. Now fix a maximal multiplicatively independent subset of $S$, say $\left(x_{1}, k_{1}\right),\left(x_{2}, k_{2}\right), \ldots,\left(x_{m}, k_{m}\right)$. Then $m \leq C$. If $m=0$ then $\# S=0 \leq C+$ $\lg C !+C \lg \lg U$, so assume $m \geq 1$.

I construct a matrix as follows. Consider the primes $q$ dividing $x_{1} x_{2} \cdots x_{m}$. The matrix has one row for each $q$, namely $\operatorname{ord}_{q} x_{1}, \operatorname{ord}_{q} x_{2}, \ldots, \operatorname{ord}_{q} x_{m}$.

The $m$ columns of this matrix are independent. Indeed, if $a_{1}, a_{2}, \ldots, a_{m}$ are integers such that $a_{1} \operatorname{ord}_{q} x_{1}+\cdots+a_{m} \operatorname{ord}_{q} x_{m}=0$ for every $q$, then $\operatorname{ord}_{q} \prod_{j} x_{j}^{a_{j}}=0$, so $\prod_{j} x_{j}^{a_{j}}=1$. The $x_{j}$ 's are independent, so every $a_{j}$ must be 0 .

Hence the matrix has $m$ independent rows. Fix $q_{1}, q_{2}, \ldots, q_{m}$ such that the corresponding rows are independent. Write

$$
Q=\left(\begin{array}{cccc}
\operatorname{ord}_{q_{1}} x_{1} & \operatorname{ord}_{q_{1}} x_{2} & \cdots & \operatorname{ord}_{q_{1}} x_{m} \\
\operatorname{ord}_{q_{2}} x_{1} & \operatorname{ord}_{q_{2}} x_{2} & \cdots & \operatorname{ord}_{q_{2}} x_{m} \\
\vdots & \vdots & \ddots & \vdots \\
\operatorname{ord}_{q_{m}} x_{1} & \operatorname{ord}_{q_{m}} x_{2} & \cdots & \operatorname{ord}_{q_{m}} x_{m}
\end{array}\right)
$$

for the matrix formed from these rows. 
By construction $\operatorname{det} Q$ is a nonzero integer. Each entry of $Q$ is bounded by $\lg U$, so $|\operatorname{det} Q| \leq m !(\lg U)^{m} \leq C !(\lg U)^{C}$.

Step 3. I claim that, for any $(x, k) \in S$ other than $\left(x_{1}, k_{1}\right), \ldots,\left(x_{m}, k_{m}\right), k$ must divide $\operatorname{det} Q$.

Let $\left(x_{0}, k_{0}\right)$ be any element of $S$ different from $\left(x_{1}, k_{1}\right), \ldots,\left(x_{m}, k_{m}\right)$. Then $k_{0}$ is coprime to $k_{1}, \ldots, k_{m}$, by hypothesis on $S$.

Since $\left(x_{1}, k_{1}\right), \ldots,\left(x_{m}, k_{m}\right)$ is a maximal multiplicatively independent subset of $S, x_{0}, x_{1}, \ldots, x_{m}$ must be multiplicatively dependent. The hypotheses of Lemma 16.1 are satisfied: each $x_{j}$ is a positive integer larger than 3. Hence there are integers $a_{0}, \ldots, a_{m}$, not all zero, with $x_{0}^{a_{0}} \cdots x_{m}^{a_{m}}=1$, and

$$
\left|a_{j}\right|<3 m^{m}\left(\log x_{0}\right) \cdots\left(\log x_{m}\right) .
$$

Since $x_{1}, \ldots, x_{m}$ are independent, $a_{0}$ must be nonzero. Without loss of generality I assume that $\operatorname{gcd}\left\{a_{0}, \ldots, a_{m}\right\}=1$; if not, divide each $a_{j}$ by the common gcd.

Suppose that $a_{0} / k_{0}+a_{1} / k_{1}+\cdots+a_{m} / k_{m} \neq 0$. Consider the matrix

$$
\Theta=\left(\begin{array}{cccc}
k_{1}+k_{0} a_{1} / a_{0} & k_{0} a_{2} / a_{0} & \cdots & k_{0} a_{m} / a_{0} \\
k_{0} a_{1} / a_{0} & k_{2}+k_{0} a_{2} / a_{0} & \cdots & k_{0} a_{m} / a_{0} \\
\vdots & \vdots & \ddots & \vdots \\
k_{0} a_{1} / a_{0} & k_{0} a_{2} / a_{0} & \cdots & k_{m}+k_{0} a_{m} / a_{0}
\end{array}\right) .
$$

By Lemma 19.1, $\Theta$ has determinant

$$
\frac{k_{0} k_{1} \cdots k_{m}}{a_{0}}\left(\frac{a_{0}}{k_{0}}+\frac{a_{1}}{k_{1}}+\cdots+\frac{a_{m}}{k_{m}}\right),
$$

which is nonzero. Hence $\Theta$ has rank $m$.

Next observe that

$$
\left(\begin{array}{c}
k_{1} \log x_{1}-k_{0} \log x_{0} \\
k_{2} \log x_{2}-k_{0} \log x_{0} \\
\vdots \\
k_{m} \log x_{m}-k_{0} \log x_{0}
\end{array}\right)=\Theta\left(\begin{array}{c}
\log x_{1} \\
\vdots \\
\log x_{m}
\end{array}\right)
$$

Indeed,

$$
\begin{aligned}
k_{i} \log x_{i}-k_{0} \log x_{0} & =k_{i} \log x_{i}+\frac{k_{0}}{a_{0}}\left(-a_{0} \log x_{0}\right) \\
& =k_{i} \log x_{i}+\frac{k_{0}}{a_{0}}\left(a_{1} \log x_{1}+\cdots+a_{m} \log x_{m}\right) .
\end{aligned}
$$

Put $B=6 m^{m}\left(\log x_{0}\right) \cdots\left(\log x_{m}\right) \max \left\{k_{j}: 0 \leq j \leq m\right\}$ and $\Omega=\prod_{1 \leq i \leq m} \log x_{i}$. Notice that

$$
B \Omega \leq 6 m^{m}(\log U)^{2 m+1} \leq 6 C^{C}(\log U)^{2 C+1} .
$$

Again the conditions of Lemma 17.1 are met: each $x_{j}$ is a positive integer; the matrix has rank $m ; x_{j} \geq 4$ and $B \geq 4$; and the matrix entries have height at most $B$. Hence, for some $i,\left|k_{i} \log x_{i}-k_{0} \log x_{0}\right| \geq \exp \left(-(16 m)^{200 m}(\Omega \log \Omega)^{1 / m} \log B \Omega\right)$. Apply Lemma 18.1 and take logarithms:

$$
\log \log (U / L)>-(16 m)^{200 m}(\Omega \log \Omega)^{1 / m} \log B \Omega .
$$


So

$$
\begin{aligned}
K(-\log \log (U / L)) & <(16 m)^{200 m} K \Omega^{1 / m}(\log \Omega)^{1 / m} \log B \Omega \\
& <(16 m)^{200 m}\left(K^{m} \prod_{1 \leq i \leq m} \log x_{i}\right)^{1 / m}(\log B \Omega)^{2} \\
& \leq(16 C)^{200 C}\left(\prod_{1 \leq i \leq m} k_{i} \log x_{i}\right)^{1 / m}(\log B \Omega)^{2} \\
& \leq(16 C)^{200 C}(\log U)\left(\log 6 C^{C}+(2 C+1) \log \log U\right)^{2} \\
& \leq K(-\log \log (U / L)) .
\end{aligned}
$$

Contradiction.

Hence $a_{0} / k_{0}+a_{1} / k_{1}+\cdots+a_{m} / k_{m}=0$. But $k_{0}$ is coprime to $k_{1}, \ldots, k_{m}$, so $k_{0}$ must divide $a_{0}$.

Consider the column vector $V=\left(a_{1}, a_{2}, \ldots, a_{m}\right)$. Since $x_{1}^{a_{1}} x_{2}^{a_{2}} \cdots x_{m}^{a_{m}}=x_{0}^{-a_{0}}$, I have $a_{1} \operatorname{ord}_{q} x_{1}+a_{2} \operatorname{ord}_{q} x_{2}+\cdots+a_{m} \operatorname{ord}_{q} x_{m}=-a_{0} \operatorname{ord}_{q} x_{0}$ for any prime $q$. In other words $a_{0}$ divides $Q V$, so $a_{0}$ divides $(\operatorname{adj} Q) Q V=(\operatorname{det} Q) V$, so $a_{0}$ divides $(\operatorname{det} Q) a_{j}$ for each $j$. But $\operatorname{gcd}\left\{a_{1}, a_{2}, \ldots, a_{m}\right\}=1$, so $a_{0}$ must $\operatorname{divide} \operatorname{det} Q$. Hence $k_{0}$ divides $\operatorname{det} Q$.

Step 4. For every pair $(x, k) \in S$, other than $\left(x_{1}, k_{1}\right), \ldots,\left(x_{m}, k_{m}\right)$, I have shown that $k$ divides $\operatorname{det} Q \neq 0$. Different pairs have coprime $k$ 's, by hypothesis, so $\operatorname{det} Q$ is divisible by the product of all those $k$ 's. Each $k$ is at least 2 . Hence there are no more than $\lg |\operatorname{det} Q| \leq \lg C !+C \lg \lg U$ pairs $(x, k)$ other than $\left(x_{1}, k_{1}\right), \ldots,\left(x_{m}, k_{m}\right)$. Finally $m \leq C$.

Lemma 19.3. Fix an interval $[L, U]$ with $U / e<L<U$ and $U \geq \exp \exp 1000$. Let $S$ be the set of primes $k$ such that there is a $k$ th power in $[L, U]$. Then

$$
\# S<(\log \log U)^{3}\left(1+\frac{\log U}{-\log \log (U / L)} \exp (30 \sqrt{\log \log U \log (2.56 \log \log U)})\right) .
$$

Proof. Define $u=\log \log U, T=(1 / 10) \sqrt{u / \log 2.56 u}$, and $C=\lfloor T\rfloor$.

Apply each piece of Lemma 18.2. First $T>1$ so $C \geq 1$ so $T<C+1 \leq 2 C$. Hence

$$
\begin{aligned}
(16 C)^{200 C}(\log U)^{1 / C} & <(16 T)^{200 T}(\log U)^{2 / T} \\
& =\exp \left(200 T \log 16 T+\frac{2 u}{T}\right)<\exp (30 \sqrt{u \log 2.56 u}) .
\end{aligned}
$$

Furthermore $6+C+\lg C !+C \lg \lg U \leq C(7+\lg C+\lg \lg U) \leq T(7+\lg T+\lg \lg U)=$ $T(7+\lg T+u / \log 2-\lg \log 2)<u^{3}$.

Set

$$
\begin{aligned}
K & =4+(16 C)^{200 C} \frac{\log U}{-\log \log (U / L)}(\log U)^{1 / C} u^{3} \\
& <4+\frac{\log U}{-\log \log (U / L)} u^{3} \exp (30 \sqrt{u \log 2.56 u}) .
\end{aligned}
$$

Now $(C+1) u<(2 T+1) u<u^{3 / 2}$ so

$$
K>(16 C)^{200 C} \frac{\log U}{-\log \log (U / L)}(\log U)^{1 / C}((C+1) u)^{2} .
$$


Furthermore,

$$
\log 6 C^{C} \leq C \log 6 C<(2 C+1) \log 6 T<(2 C+1) u \leq(2 T+1) u<(1 / 2) u^{3 / 2},
$$

SO

$$
\begin{aligned}
K & >(16 C)^{200 C} \frac{\log U}{-\log \log (U / L)}(\log U)^{1 / C}\left(\log 6 C^{C}+(2 C+1) u\right)^{2} \\
& \geq(16 C)^{200 C} \frac{\log U}{-\log \log (U / L)}\left(\log 6 C^{C}+(2 C+1) u\right)^{2} .
\end{aligned}
$$

Finally I count the primes $k \in S$. There are at most $K-1$ primes $k<K$. For each $k \geq K$ select an integer $x$ such that $x^{k} \in[L, U]$. Consider the pairs $(x, k)$. By Lemma 19.2, there are at most $C+\lg C !+C \lg \lg U$ pairs with $x \geq 4$. Since $U / L<3$, there is at most one power of 3 in $[L, U]$, and at most two powers of 2 . Hence

$$
\begin{aligned}
\# S & \leq K+2+C+\lg C !+C \lg \lg U \\
& <u^{3}\left(1+\frac{\log U}{-\log \log (U / L)} \exp (30 \sqrt{\log \log U \log 2.56 u})\right)
\end{aligned}
$$

as desired.

Lemma 19.4. Fix $n \geq \exp \exp$ 1000. Set $u=\log \log 2 n$. Fix $v$ with $1 \leq v \leq$ $\log _{5} n$. Let $S$ be the set of primes $k$ such that there is a $k$ th power in the interval $\left[n-n^{1-1 / v}, n+n^{1-1 / v}\right]$. Then $\# S<3 v u^{3} \exp (30 \sqrt{u \log 2.56 u})$.

Proof. Set $L=n-n^{1-1 / v}$ and $U=n+n^{1-1 / v}<2 n$. By Lemma 18.3, $\log U<$ $-2 v \log \log (U / L)$. Also $U>n \geq \exp \exp 1000$, and $U / L \leq(1+1 / 5) /(1-1 / 5)<e$, so $\# S<(\log \log U)^{3}(1+2 v \exp (30 \sqrt{\log \log U \log (2.56 \log \log U}))$ by Lemma 19.3. Finally $\log \log U<u$.

Corollary 19.5. For $n \geq 16$, there are fewer than $\exp (40 \sqrt{\log \log n \log \log \log n})$ perfect powers in the interval $[n, n+\sqrt{n}]$.

Proof. Let $S$ be the set of primes $p$ such that there is a $p$ th power in $I=[n, n+\sqrt{n}]$. Each perfect power in $I$ is a $p$ th power for some prime $p$. On the other hand, $I$ is too short to contain two $p$ th powers: if $x^{p} \geq n$ then $(x+1)^{p} \geq x^{p}+p x^{p-1} \geq$ $n+p n^{1-1 / p}>n+\sqrt{n}$. Hence the number of perfect powers in $I$ is at most $\# S$.

Write $t=\log \log n$. I will show that $\# S<\exp (40 \sqrt{t \log t})$. For $t<1000$ this is easy. If $p \in S$ then $p \leq \lg (n+\sqrt{n})<\lg 2 n$. So $\# S<\lg n=\exp (t-\log \log 2)<$ $\exp (40 \sqrt{t \log t})$ by Lemma 18.4 .

For $t \geq 1000$, apply Lemma 19.4 with $v=2$. Set $u=\log \log 2 n$. Then $\# S<$ $6 u^{3} \exp (30 \sqrt{u \log 2.56 u})$. Finally, by Lemma 18.5, $\# S<\exp (40 \sqrt{t \log t})$.

Notes. Corollary 19.5 was stated in [20, Theorem 1]. There is a gap in the proof in [20]: it incorrectly assumes that, in my notation, $a_{0} / k_{0}+a_{1} / k_{1}+\cdots+a_{m} / k_{m} \neq 0$. (Note that " $-a_{m+1} b_{j} / b_{m+1}$ " in [20] was a typo for " $+a_{m+1} b_{j} / b_{m+1} . "$ )

John Loxton has closed the gap, and has graciously allowed me to present his correction here. His idea is expressed above in Step 2 and Step 4 of Lemma 19.2. Other than this, the approach here is the same as the approach of [20, Theorem 1], modified slightly to handle more general intervals $[L, U]$.

The conclusion of Lemma 19.2 could easily be improved. Each column of the matrix $Q$ has sum at most $(\lg U) / K$. From this one can prove with Hadamard's 
inequality [15, exercise 4.6.1-15] or with Gershgorin's inequality-see [13, problem $6.1-3]$ - that the determinant of $Q$ is at most $((\lg U) / K)^{m}$.

In general the bounds in this section are very far from best possible. A more careful study would produce many quantitative improvements and perhaps some qualitative improvements.

Let $S$ be the set of exponents $k$ such that there is a $k$ th power in $[L, U]$. One could prove a bound on the size of $S$ as follows. Lemma 19.3 supplies a bound - call it $m$-on the number of primes in $S$. Every $k \in S$ is built up from those primes. Hence the size of $S$ is at most the number of products $\leq \lg U$ of those primes, which is at most the number of products $\leq \lg U$ of the first $m$ prime numbers, which in turn can be estimated by analytic techniques. See [28] and [10].

\section{Final $F(n)$ ANALYSis}

In this section, I use Lemma 19.4 to bound the function $F(n)$ introduced in section 12. This upper bound is in $(\lg n)^{1+o(1)}$.

Lemma 20.1. Fix $n \geq \exp \exp 1000$. Set $u=\log \log 2 n$. Then

$$
F(n)<(\lg n \lg \lg n)\left(1+3 u^{3} \exp (30 \sqrt{u \log 2.56 u}) \lg (4 \lg n)\right) .
$$

Proof. Write $g(p)=\left(\text { round } n^{1 / p}\right)^{p}$. Also abbreviate $K=3 u^{3} \exp (30 \sqrt{u \log 2.56 u})$.

The critical idea here is to sort the primes $p$ by $d(n, g(p))$. Let $c<\lg n$ be the number of primes between 2 and $\lg n$. Let $p_{1}, p_{2}, \ldots, p_{c}$ be the primes, in such an order that $d\left(n, g\left(p_{j}\right)\right)$ is a nondecreasing function of $j$.

Now $F(n)=\sum_{1 \leq j \leq c}\left(\lg p_{j}\right) \max \left\{1, \lg n-d\left(n, g\left(p_{j}\right)\right)\right\}$. I estimate this sum in two pieces: first where $1 \leq j<K$, second where $K \leq j \leq c$.

There are fewer than $K$ terms in the first piece, and each term is less than $\lg n \lg \lg n$, so the sum of the terms in the first piece is less than $K \lg n \lg \lg n$.

In the second piece, set $v=j / K \geq 1$. I have $j \leq c<\lg n$ and $K>3$ so $v<\log _{5} n$.

Suppose that $\lg n-d\left(n, g\left(p_{j}\right)\right)>1+(1 / v) \lg n$. Then

$$
\left|n-g\left(p_{i}\right)\right|<2^{d\left(n, g\left(p_{i}\right)\right)+1} \leq 2^{d\left(n, g\left(p_{j}\right)\right)+1}<2^{(1-1 / v) \lg n}=n^{1-1 / v}
$$

for all $i \leq j$. So there is a $p_{i}$ th power within $n^{1-1 / v}$ of $n$ for $1 \leq i \leq j$. But that is impossible, since by Lemma 19.4 there are fewer than $K v=j$ primes $p$ with a $p$ th power so close to $n$.

Hence $\lg n-d\left(n, g\left(p_{j}\right)\right) \leq 1+(1 / v) \lg n$. So the sum of this piece is at most $\sum_{K \leq j \leq c}(\lg \lg n)(1+(K / j) \lg n)<(\lg \lg n) \sum_{1 \leq j \leq c}(1+(K / j) \lg n)<c \lg \lg n+$ $K \lg n \lg \lg n \lg 2 c$.

Notes. Various constants here can of course be improved.

\section{PART VI. PRACTICAL IMPROVEMENTS}

\section{The 2-ADIC VARIANT}

In this section, I describe a 2-adic variant of Algorithm X. With this variant, I can work with integers rather than floating-point numbers; I no longer need guard bits; I can jump directly into Newton's method without a preliminary binary search; and a proper error analysis takes a few lines rather than several pages.

It will be convenient to restrict attention to odd $n$. See section 22 for a method to handle even $n$. 
Motivation. To check if $n$ is a $k$ th power, I compute a tentative $k$ th root of $n$-an integer $x$ such that no integer other than $x$ can possibly be the $k$ th root of $n$. Then I test whether $x^{k}=n$.

To find $x$ in Part II and Part III, I constructed a number that was close to a $k$ th root of $n$ in the usual metric. I used the same metric again to check whether $x^{k}=n$ : I computed $x^{k}$ in low precision to see whether it was close to $n$.

Nothing in the original problem suggests this metric. The 2 -adic variant uses a different metric, where $i$ and $j$ are close if $i-j$ is divisible by a high power of 2 .

Notation. This section deviates from the notation of Parts I, II, and III: $r, y$, and $z$ are odd integers rather than positive floating-point numbers.

Lemma 21.1. If $2 i \equiv 2 j\left(\bmod 2^{b+1}\right)$ and $b \geq 1$ then $i^{2} \equiv j^{2}\left(\bmod 2^{b+1}\right)$.

2-adic approximate powers. Fix positive integers $k$ and $b$. For any integer $m$ define $\operatorname{pow}_{2, b}(m, k)=m^{k} \bmod 2^{b}$. See section 6 for methods of computing $\operatorname{pow}_{2, b}(m, k)$ without many multiplications. As I compute $\operatorname{pow}_{2, b}(m, k)$, I keep track of an "overflow bit" to figure out whether $m^{k} \bmod 2^{b}=m^{k}$.

Checking tentative $k$ th roots. Here is a straightforward algorithm for checking whether $x^{k}=n$.

Algorithm C2. Given positive integers $n, x, k$, to see if $n=x^{k}$ : In advance set $f=\lfloor\lg 2 n\rfloor$.

1. If $x=1$ : Print 0 if $n=1$. Print 2 if $n \neq 1$. Stop.

2. Set $b \leftarrow 1$.

3. Compute $r \leftarrow \operatorname{pow}_{2, b}(x, k)$. Simultaneously figure out if $r=x^{k}$.

4. If $n \bmod 2^{b} \neq r$, print 2 and stop.

5. If $b \geq f$ : Print 0 if $r=x^{k}$. Print 2 if $r \neq x^{k}$. Stop.

6 . Set $b \leftarrow \min \{2 b, f\}$. Go back to step 3 .

Lemma 21.2. Algorithm C2 prints 0 if and only if $n=x^{k}$.

Proof. If $n=x^{k}$ then $r=\operatorname{pow}_{2, b}(x, k)=x^{k} \bmod 2^{b}=n \bmod 2^{b}$ so Algorithm $\mathrm{C}$ never stops in step 4 . Hence it stops in step 5 . When it does, $b \geq f$, so $r=n \bmod 2^{f}=n=x^{k}$. Thus it prints 0 . Conversely, if it prints 0 , then $x^{k}=r=$ $n \bmod 2^{f}=n$.

2-adic approximate multiplication and division. Fix $b \geq 1$. For $m$ an integer and $k$ a positive integer write $\operatorname{mul}_{2, b}(m, k)=k m \bmod 2^{b}$.

If $k$ is odd, write $\operatorname{div}_{2, b}(m, k)$ for the unique integer between 0 inclusive and $2^{b}$ exclusive such that $m \equiv k \operatorname{div}_{2, b}(m, k)\left(\bmod 2^{b}\right)$.

Finding 2-adic approximate $k$ th roots. Fix an odd integer $y$ and a positive odd integer $k$. I will find an approximate negative $k$ th root of $y$ by Newton's method. For motivation see section 8. (Question: Why do I insist that $k$ be odd? Answer: Square roots introduce a bit of difficulty. See Algorithm S2 below.) 
For each $b \geq 1$, I define and construct an odd integer $\operatorname{nroot}_{2, b}(y, k)$, between 0 and $2^{b}$, by the following algorithm:

Algorithm N2. Given an odd integer $y$ and positive integers $b, k$ with $k$ odd, to compute $\operatorname{nroot}_{2, b}(y, k)$ : In advance set $b^{\prime}=\lceil b / 2\rceil$.

1. If $b=1: \operatorname{nroot}_{2, b}(y, k)=1$. Stop.

2. Compute $z \leftarrow \operatorname{nroot}_{2, b^{\prime}}(y, k)$ by Algorithm N2.

3. Set $r_{2} \leftarrow \operatorname{mul}_{2, b}(z, k+1)$.

4. Set $r_{3} \leftarrow y \operatorname{pow}_{2, b}(z, k+1) \bmod 2^{b}$.

5. Set $r_{4} \leftarrow \operatorname{div}_{2, b}\left(r_{2}-r_{3}, k\right)$. Now $\operatorname{nroot}_{2, b}(y, k)=r_{4}$.

Lemma 21.3. If $k$ is odd and $r=\operatorname{nroot}_{2, b}(y, k)$ then $r^{k} y \bmod 2^{b}=1$.

Proof. If $b=1$ then $r=1$ and $y \bmod 2=1$.

If $b \geq 2$ then $r$ shows up as $r_{4}$ in Algorithm N2. Note that $b^{\prime}<b$. By induction $z^{k} y \bmod 2^{b^{\prime}}=1$. So $z^{k} y=1+2^{b^{\prime}} j$ for some integer $j$.

Note that $2^{2 b^{\prime}} \equiv 0\left(\bmod 2^{b}\right)$. So $\left(k-2^{b^{\prime}} j\right)^{k} \equiv k^{k}-k 2^{b^{\prime}} j k^{k-1}=k^{k}\left(1-2^{b^{\prime}} j\right)$ by the binomial theorem.

By construction $r_{2} \equiv(k+1) z, r_{3} \equiv z^{k+1} y$, and $k r_{4} \equiv r_{2}-r_{3}$. Hence $k r_{4} \equiv$ $z\left(k+1-z^{k} y\right)=z\left(k-2^{b^{\prime}} j\right)$. So

$$
k^{k} r_{4}^{k} y \equiv z^{k} y\left(k-2^{b^{\prime}} j\right)^{k} \equiv\left(1+2^{b^{\prime}} j\right) k^{k}\left(1-2^{b^{\prime}} j\right) \equiv k^{k}\left(1-2^{2 b^{\prime}} j^{2}\right) \equiv k^{k} .
$$

But $k^{k}$ is odd, so $r^{k} y=r_{4}^{k} y \equiv 1\left(\bmod 2^{b}\right)$ as claimed.

Finding 2-adic approximate square roots. Again fix an odd integer $y$. For each $b \geq 1$, I define and construct $\operatorname{nroot}_{2, b}(y, 2)$ by the following algorithm:

Algorithm S2. Given an odd integer $y$ and a positive integer $b$, to compute an integer $\operatorname{nroot}_{2, b}(y, 2)$ : In advance set $b^{\prime}=\lceil(b+1) / 2\rceil$.

1. If $b=1: \operatorname{nroot}_{2, b}(y, 2)$ is 1 if $y \bmod 4=1,0$ otherwise. Stop.

2. If $b=2: \operatorname{nroot}_{2, b}(y, 2)$ is 1 if $y \bmod 8=1,0$ otherwise. Stop.

3. Compute $z \leftarrow \operatorname{nroot}_{2, b^{\prime}}(y, 2)$ by Algorithm S2.

4. If $z=0: \operatorname{nroot}_{2, b}(y, 2)=0$. Stop.

5 . Set $r_{2} \leftarrow \operatorname{mul}_{2, b+1}(z, 3)$.

6. Set $r_{3} \leftarrow y$ pow $_{2, b+1}(z, 3) \bmod 2^{b+1}$.

7. Set $r_{4} \leftarrow\left(r_{2}-r_{3}\right) / 2 \bmod 2^{b}$. Now $\operatorname{nroot}_{2, b}(y, 2)=r_{4}$.

Lemma 21.4. Set $r=\operatorname{nroot}_{2, b}(y, 2)$. If $i^{2} y \bmod 2^{b+1}=1$ for some odd integer $i$ then $r \neq 0$. If $r \neq 0$ then $r^{2} y \bmod 2^{b+1}=1$.

Proof. First consider $b=1$. If $y \bmod 4=1$ then $r=1$ so $r^{2} y \bmod 4=1$. If $y \bmod 4=3$ then $r=0$ and $i^{2} y \bmod 4=3$ for any $i$.

Next consider $b=2$. If $y \bmod 8=1$ then $r=1$ so $r^{2} y \bmod 8=1$. If $y \bmod 8 \neq 1$ then $r=0$ and $i^{2} y \bmod 8=y \bmod 8 \neq 1$ for any $i$.

If $b \geq 3$ then $r$ shows up as $r_{4}$ in Algorithm S2. Note that $b^{\prime}<b$. If $z=0$ then by induction $i^{2} y \bmod 2^{b^{\prime}+1}$ is never 1 , so $i^{2} y \bmod 2^{b+1}$ is never 1 ; and $r=0$.

If $z \neq 0$ then by induction $z^{2} y \bmod 2^{b^{\prime}+1}=1$. So $z^{2} y=1+2^{b^{\prime}+1} j$ for some integer $j$. Note that $\left(1-2^{b^{\prime}} j\right)^{2}=1-2^{b^{\prime}+1} j+2^{2 b^{\prime}} j^{2} \equiv 1-2^{b^{\prime}+1} j\left(\bmod 2^{b+1}\right)$ since $2 b^{\prime} \geq b+1$. 
By construction $r_{2} \equiv 3 z\left(\bmod 2^{b+1}\right), r_{3} \equiv z^{3} y$, and $2 r_{4} \equiv r_{2}-r_{3}$. Hence $2 r_{4} \equiv z\left(3-z^{2} y\right)=z\left(2-2^{b^{\prime}+1} j\right)$. By Lemma $21.1, r_{4}^{2} \equiv z^{2}\left(1-2^{b^{\prime}} j\right)^{2}$. Thus

$$
r^{2} y=r_{4}^{2} y \equiv z^{2} y\left(1-2^{b^{\prime}} j\right)^{2} \equiv\left(1+2^{b^{\prime}+1} j\right)\left(1-2^{b^{\prime}+1} j\right)=1-2^{2 b^{\prime}+2} j^{2} \equiv 1
$$

as claimed.

Perfect-power decomposition. I imitate Algorithm K from section 10: to see if $n$ is a $k$ th power, I compute and then check a tentative $k$ th root.

Algorithm K2. Given a positive odd integer $n$, an integer $k \geq 2$ such that either $k=2$ or $k$ is odd, and an odd integer $y$ (see Lemma 21.5), to see if $n$ is a $k$ th power: In advance set $f=\lfloor\lg 2 n\rfloor$ and $b=\lceil f / k\rceil$.

1. Calculate $r \leftarrow \operatorname{nroot}_{2, b}(y, k)$.

2. If $k=2$ : If $r=0$, print 0 and stop.

3. Check if $n=r^{k}$ with Algorithm C2. If so, print $r$ and stop.

4. If $k=2$ : Check if $n=\left(2^{b}-r\right)^{k}$ with Algorithm C2. If so, print $2^{b}-r$ and stop. 5. Print 0 and stop.

Lemma 21.5. Set $f=\lfloor\lg 2 n\rfloor$ and $b=\lceil f / k\rceil$. Assume that yn $\bmod 2^{b+1}=1$. If $n$ is a kth power, Algorithm K2 prints $n^{1 / k}$. If $n$ is not a kth power, Algorithm K2 prints 0 .

Proof. Case 1: $n$ is not a $k$ th power. Then $n \neq r^{k}$ and $n \neq\left(2^{b}-r\right)^{k}$, so Algorithm K2 does not stop in steps 3 or 4 . So it prints 0 .

Case 2: $n=x^{k}$, and $k$ is odd. By Lemma 21.3, $r^{k} y \bmod 2^{b}=1$. Furthermore $y n \bmod 2^{b}=1$ so $r^{k} \equiv n=x^{k}\left(\bmod 2^{b}\right)$.

Put $c=r^{k-1}+r^{k-2} x+\cdots+x^{k-1}$; each term in this sum is odd, and there are $k$ terms, so $c$ is odd. But $2^{b}$ divides $r^{k}-x^{k}=(r-x) c$ so $2^{b}$ divides $r-x$. Both $r$ and $x$ are positive integers smaller than $2^{b}$, so $r=x$. Hence $n=r^{k}$; Algorithm K prints $r$ in step 3 .

Case 3: $n=x^{k}$, and $k=2$. By Lemma 21.4, $r$ is nonzero, since $y x^{2} \bmod 2^{b+1}=$ 1. By Lemma 21.4 again, $r^{2} y \bmod 2^{b+1}=1$. Hence $r^{2} \equiv x^{2}\left(\bmod 2^{b+1}\right)$.

Either $r \equiv x(\bmod 4)$ or $r \equiv-x(\bmod 4)$. Say $r \equiv x$; then $r+x \equiv 2$. Now $2^{b+1}$ divides $r^{2}-x^{2}=(r-x)(r+x)$, and only one power of 2 divides $r+x$, so $r \equiv x\left(\bmod 2^{b}\right)$. Both $r$ and $x$ are positive integers smaller than $2^{b}$, so $r=x$, so Algorithm K prints $r$ in step 3 .

If $r \equiv-x(\bmod 4)$ then $r \neq x$ so $r^{2} \neq n$ so Algorithm $\mathrm{K}$ does not print $r$ in step 3 . However, $2^{b}-r \equiv x(\bmod 4)$, and $2^{b+1}$ divides $\left(2^{b}-r\right)^{2}-x^{2}$, so as above $2^{b}-r \equiv x\left(\bmod 2^{b}\right)$. Thus $2^{b}-r=x$ and Algorithm $\mathrm{K}$ prints $2^{b}-r$ in step 4.

Algorithm X2. Given an odd integer $n \geq 2$, to decompose $n$ as a perfect power if possible: In advance set $f=\lfloor\lg 2 n\rfloor$.

1. Compute $y \leftarrow \operatorname{nroot}_{2,\lceil f / 2\rceil+1}(n, 1)$.

2. For each prime number $p<f$ :

3. Apply Algorithm K2 to $(n, p, y)$; let $x$ be the result.

4. If $x>0$, print $(x, p)$ and stop.

5. Print $(n, 1)$.

Lemma 21.6. If $n$ is a perfect power, Algorithm X2 prints a prime number $p$ and a positive integer $x$ such that $x^{p}=n$. If $n$ is not a perfect power, Algorithm X2 prints $(n, 1)$. 
Proof. By Lemma 21.3, yn mod $2^{\lceil f / 2\rceil+1}=1$. If Algorithm X2 stops in step 4 then $x^{p}=n$ by Lemma 21.5. If Algorithm X2 never stops in step 4 then, by Lemma $21.5, n$ is not a $p$ th power for any prime $p<f$, so $n$ is not a perfect power.

I could synthesize Algorithm X and Algorithm X2. For each $k$, I can compute a tentative $k$ th root $x$ by either Algorithm $\mathrm{N}$ or Algorithm N2; I can then check whether $x^{k}=n$ by either Algorithm C or Algorithm C2. After Algorithm N it is probably best to try Algorithm C2 first; after Algorithm N2 it is probably best to try Algorithm $\mathrm{C}$ first. I could run Algorithm $\mathrm{C}$ and Algorithm $\mathrm{C} 2$ in parallel, stopping as soon as either algorithm sees that $n \neq x^{k}$.

It is possible to convert $n$ into base $q$ for $q>2$, and then use the $q$-adics instead of the 2-adics. This is probably not worthwhile in practice, unless for some strange reason $n$ is already known in base $q$. But it may be worthwhile to compute $n \bmod q$. See the next section for further discussion.

Notes. See [15, exercise 4.1-31] for an introduction to the 2-adic numbers.

Two $q$-adic applications of Newton's method are generally known as "Hensel's lemma." The first is the use of Newton's method to refine a $q$-adic root of a polynomial; see [30, page 14] or [12, page 84]. The second is the more general use of Newton's (multidimensional) method to refine a $q$-adic factor of a polynomial; see [15, exercise 4.6.2-22], [22, Theorem 8.3], or [25, page 40].

See [15, exercise 4.4-14] or [8] for a fast method of converting $n$ into base $q$.

My previous perfect-power run-time analysis does not apply if I use Algorithm $\mathrm{C} 2$ in place of Algorithm C. The results of Part IV would remain valid, but to prove that the resulting perfect-power detection algorithm runs in essentially linear time I would need 2-adic versions of the theorems in Part V, and in particular of [20, Theorem 4].

\section{TRIAL DIVISION}

As usual fix $n \geq 2$. In this section I discuss several tricks based on computing $n \bmod q$ for one or more primes $q$.

If $n$ has no small prime divisors, lower the exponent bound. If $n$ is odd and $n=x^{k}$ then $x$ is also odd. So $x \geq 3$ and $k \leq \log _{3} n$. More generally one may compute $n \bmod q$ for all primes $q<T$, for some bound $T$. If $n \bmod q$ is always nonzero, one need not check exponents past $\log _{T} n$.

If $n$ has a prime divisor, find its order. What if $n \bmod q=0$ ? First compute the number $\operatorname{ord}_{q} n$ of factors $q$ in $n$, together with $n / q^{\operatorname{ord}_{q} n}$. Then check, for each $p$ dividing $\operatorname{ord}_{q} n$, whether $n / q^{\operatorname{ord}_{q} n}$ is a $p$ th power. Otherwise $n$ cannot be a perfect power. (Note that $n / q^{\operatorname{ord}_{q} n}$ may be 1 , in which case no testing is necessary.)

Recall that the 2 -adic method in section 21 requires that $n$ be odd. This is not a serious restriction. If $n$ is even, the method here ends up checking whether $n / 2^{\operatorname{ord}_{2} n}$ is a $p$ th power, for various primes $p$; and $n / 2^{\operatorname{ord}_{2} n}$ is odd.

There are several plausible ways to compute the number of factors $q$ in $n$.

If $q=2$ then $\operatorname{ord}_{q} n$ is the number of 0 bits at the bottom of $n$ 's binary expansion.

If $q>2$, I do a binary search upon the number of factors. The idea is to compute $n \bmod q^{c}$ and $\left\lfloor n / q^{c}\right\rfloor$ for some integer $c \approx\left(\log _{q} n\right) / 2$. If $n \bmod q^{c} \neq 0$ then $\operatorname{ord}_{q} n=\operatorname{ord}_{q}\left(n \bmod q^{c}\right)$; if $n \bmod q^{c}=0$ then $\operatorname{ord}_{q} n=c+\operatorname{ord}_{q}\left(n / q^{c}\right)$. 
Chop $c$ in half and repeat. This method takes essentially linear time given fast multiplication and the algorithms from section 8 .

I could instead do a linear search; this amounts to always taking $c=1$ in the above description. This will be faster than a binary search on average. I could compromise with a sequence of $c$ that is at first optimistic but backs off quickly if necessary. For example: begin with $c=1$, double $c$ if $n \bmod q^{c}=0$, and chop $c$ in half if $n \bmod q^{c} \neq 0$.

Check the character of residues of $n$. If $n$ is a $k$ th power, and $q$ is a prime with $q \bmod k=1$ then $n^{(q-1) / k} \bmod q$ is either 0 or 1 . A non- $k$ th power has roughly a $1 / k$ chance of passing this test.

Check the residues of tentative roots. If $n=x^{k}$ then $n \bmod q=x^{k} \bmod q$. So, given $n \bmod q$, I can try to weed out a tentative root $x$ by calculating the $k$ th power modulo $q$ of $x \bmod q$. In practice this test is quite powerful: if $n \neq x^{k}$ then very few primes $q$ divide $n-x^{k}$.

In this test $q$ need not be prime. It might be convenient to check whether $n$ agrees with $x^{k}$ modulo $2^{32}$, for example, although this is redundant if $x$ was constructed by 2 -adic methods.

One could develop a fast randomized power-testing algorithm along these lines. Start from a tentative root $x$. First check if $x^{k} \leq 2 n$. Then check if $n \bmod q$ equals $x^{k} \bmod q$ for a set of "random" primes $q$ with product larger than $n$. This test will succeed if and only if $n=x^{k}$. If $n \neq x^{k}$ then one will, on average, test very few $q$ 's.

Check for small divisors of tentative roots. If $n$ is not divisible by any primes $q<T$, and $n=x^{k}$ then $x$ is not divisible by any primes $q<T$. So I can throw away any tentative root $x$ that has prime factors smaller than $T$. This is much weaker than testing whether $n \bmod q=x^{k} \bmod q$ for each $q<T$, but it is also much faster.

Notes. See generally [4] for precedents. The approach of [4] is, for each $k$, to precompute a database of primes $q$ with $q$ mod $k=1$, and then to systematically compute the characters $n^{(q-1) / k} \bmod q$ for each $q$ in the database. [4] also suggests (1) checking whether $n$ is divisible by small primes, (2) lowering the exponent bound if $n$ is not divisible by any small primes, and (3) finding $\operatorname{ord}_{q} n$ (with a linear search) if $q$ divides $n$.

My binary search method for computing $\operatorname{ord}_{q} n$ is a straightforward optimization of the following procedure: first apply [15, exercise 4.4-14] to write $n$ in base $q$; then see how many of the low "qits" are zero.

The best way to compute $n \bmod q$ for many $q$ simultaneously is by binary splitting; see, e.g., [1, page 291].

See [19] for an overview of practical and theoretical methods for checking whether $x$ (or $n$ ) has a prime factor smaller than $T$.

In some applications one may know $n \bmod q$, or a representation of $n$ from which $n \bmod q$ is easy to derive. Victor Miller points out that if $n$ is represented in the factorial base [15, equation 4.1-10] then it is easy to compute $n \bmod q$ for small primes $q$.

I have many options here. Each subset of options poses a new optimization problem - e.g., if I use characters as in [4] but with fast arithmetic and binary splitting, how much trial division should I do? - for which an exact answer will 
depend heavily on characteristics of the computer at hand. Having not yet solved all such problems, I do not feel competent to declare one algorithm the "winner."

\section{REFERENCES}

1. Alfred V. Aho, John E. Hopcroft, Jeffrey D. Ullman, The design and analysis of computer algorithms, Addison-Wesley, Reading, Massachusetts, 1974. MR 54:1706

2. Robert S. Anderssen and Richard P. Brent (editors), The complexity of computational problem solving, University of Queensland Press, Queensland, 1976.

3. Eric Bach, Jeffrey Shallit, Algorithmic number theory, MIT Press, Boston, Massachusetts, 1996. MR 97e: 11157

4. Eric Bach, Jonathan Sorenson, Sieve algorithms for perfect power testing, Algorithmica 9 (1993), 313-328. MR 94d:11103

5. Alan Baker, The theory of linear forms in logarithms, in [6], pp. 1-27. MR 58:16543

6. Alan Baker, David William Masser (editors), Transcendence theory: advances and applications, Academic Press, 1977. MR 56:15573

7. Jonathan M. Borwein, Peter B. Borwein, Pi and the AGM, Wiley, New York, 1987. MR 89a:11134

8. Richard P. Brent, The complexity of multiple-precision arithmetic, in [2], pp. 126-165.

9. Richard P. Brent, Fast multiple-precision evaluation of elementary functions, Journal of the Association for Computing Machinery 23 (1976), 242-251. MR 52:16111

10. E. Rodney Canfield, Paul Erdős, Carl Pomerance, On a problem of Oppenheim concerning "factorisatio numerorum", Journal of Number Theory 17 (1983), 1-28. MR 85j:11012

11. Henri Cohen, A course in computational algebraic number theory, Springer-Verlag, Berlin, 1993. MR 94i: 11105

12. Albrecht Fröhlich, Martin J. Taylor, Algebraic number theory, Cambridge University Press, Cambridge, 1991. MR 94d:11078

13. Roger A. Horn, Charles A. Johnson, Matrix analysis, Cambridge University Press, Cambridge, 1985. MR 87e: 15001

14. Donald E. Knuth, The art of computer programming, volume 1: fundamental algorithms, 2nd edition, Addison-Wesley, Reading, Massachusetts, 1973. MR 51:14624

15. Donald E. Knuth, The art of computer programming, volume 2: seminumerical algorithms, 2nd edition, Addison-Wesley, Reading, Massachusetts, 1981. MR 83i:68003

16. Arjen K. Lenstra, Hendrik W. Lenstra, Jr. (editors), The development of the number field sieve, Lecture Notes in Mathematics 1554, Springer-Verlag, Berlin, 1993. MR 96m:11116

17. Arjen K. Lenstra, Hendrik W. Lenstra, Jr., Mark S. Manasse, John M. Pollard, The number field sieve, in [16], pp. 11-40. MR 96m:11116

18. Hendrik W. Lenstra, Jr., private communication.

19. Hendrik W. Lenstra, Jr., Jonathan Pila, Carl Pomerance, A hyperelliptic smoothness test. I, Philosophical Transactions of the Royal Society of London, Series A 345 (1993), 397-408. MR 94m: 11107

20. John H. Loxton, Some problems involving powers of integers, Acta Arithmetica 46 (1986), 113-123. MR 87j:11071

21. John H. Loxton, Alfred J. van der Poorten, Multiplicative dependence in number fields, Acta Arithmetica 42 (1983), 291-302. MR 86b:11052

22. Hideyuki Matsumura, Commutative ring theory, Cambridge University Press, Cambridge, 1986. MR 88h:13001

23. James Munkres, Elements of algebraic topology, Addison-Wesley, Reading, Massachusetts, 1984. MR 85m:55001

24. Henri J. Nussbaumer, Fast polynomial transform algorithms for digital convolution, IEEE Transactions on Acoustics, Speech, and Signal Processing 28 (1980), 205-215. MR 80m:94004

25. Michael E. Pohst, Computational algebraic number theory, Birkhäuser, Basel, 1993. MR 94j:11132

26. William H. Press, Brian P. Flannery, Saul P. Teukolsky, William P. Vetterling, Numerical recipes: the art of scientific computing, Cambridge University Press, Cambridge, 1986. MR 87m:65001a

27. Paul Pritchard, Fast compact prime number sieves (among others), J. Algorithms 4 (1983), 332-344. MR 85h:11080 
28. J. Barkley Rosser, Lowell Schoenfeld, Approximate formulas for some functions of prime numbers, Illinois Journal of Mathematics 6 (1962), 64-94. MR 25:1139

29. Arnold Schönhage, Volker Strassen, Schnelle Multiplikation großer Zahlen, Computing 7 (1971), 281-292. MR 45:1431

30. Jean-Pierre Serre, A course in arithmetic, Springer-Verlag, New York, 1973. MR 49:8956

31. E. T. Whittaker, G. N. Watson, A course of modern analysis, 4th edition, Cambridge University Press, 1927. MR 31:2375 (1962 reprint)

Department of Mathematics, University of California, Berkeley, California 94720

Current address: Department of Mathematics, Statistics, and Computer Science, The University of Illinois at Chicago, Chicago, Illinois 60607-7045

E-mail address: djb@pobox.com 